\title{
Flaperon Modification Effect on Jet-Flap Interaction Noise Reduction for Chevron Nozzles
}

\author{
Vinod G. Mengle*, Robert W. Stoker ${ }^{\dagger}$, Leon Brusniak ${ }^{*}$ and Ronen Elkoby ${ }^{\S}$ \\ The Boeing Company, Seattle, WA, 98124-2207 \\ and \\ Russell H. Thomas ${ }^{* *}$ \\ NASA Langley Research Center, Hampton, VA, 23681-2199
}

\begin{abstract}
Jet-flap interaction (JFI) noise can become an important component of far field noise when a flap is immersed in the engine propulsive stream or is in its entrained region, as in approach conditions for under-the-wing engine configurations. We experimentally study the effect of modifying the flaperon, which is a high speed aileron between the inboard and outboard flaps, at both approach and take-off conditions using scaled models in a free jet. The flaperon modifications were of two types: sawtooth trailing edge and mini vortex generators (vg's). Parametric variations of these two concepts were tested with a round coaxial nozzle and an advanced chevron nozzle, with azimuthally varying fan chevrons, using both far field microphone arrays and phased microphone arrays for source diagnostics purposes. In general, the phased array results corroborated the far field results in the upstream quadrant pointing to JFI near the flaperon trailing edge as the origin of the far field noise changes. Specific sawtooth trailing edges in conjunction with the round nozzle gave marginal reduction in JFI noise at approach, and parallel co-rotating mini-vg's were somewhat more beneficial over a wider range of angles, but both concepts were noisier at take-off conditions. These two concepts had generally an adverse JFI effect when used in conjunction with the advanced chevron nozzle at both approach and take-off conditions.
\end{abstract}

\section{Nomenclature}

$\begin{array}{ll}\text { AVC } & =\text { azimuthally varying chevrons } \\ \mathbf{B} & =\text { baseline flaperon } \\ I & =\text { installed noise } \\ J F I & =\text { jet/flap interaction } \\ \mathbf{L} & =\text { longer sawtooth flaperon (lower aspect ratio) } \\ \mathbf{L}, & =\text { longer sawtooth flaperon with rounded tips } \\ L S A F & =\text { Low Speed Aeroacoustic Facility (at Boeing) } \\ M & =\text { Mach number } \\ \mathbf{N} & =\text { flaperon with more number of sawteeth than R-type of flaperon } \\ N P R & =\text { nozzle pressure ratio, nozzle/ambient } \\ p & =\text { acoustic pressure } \\ Q & =\text { dipole strength } \\ \mathbf{R} & =\text { reference sawtooth flaperon; regular/reference chevrons } \\ \mathbf{S} & =\text { sawtooth flaperon with rounded tips and valleys } \\ S & =\text { measured installed noise (log sum of true installed noise and tunnel noise) }\end{array}$

\footnotetext{
${ }^{*}$ Engineer, Acoustics \& Fluid Mechanics Technology, P.O. Box 3707, MC: 67-ML. Sr. AIAA Member.

${ }^{\dagger}$ Engineer, 787 Community Noise, P.O. Box 3707, MC: 02-XL, Sr. AIAA Member.

* Engineer, Acoustics \& Fluid Mechanics Technology, P.O. Box 3707, MC: 67-ML..

${ }^{\S}$ Engineer, Acoustics Technology, Phantom Works, MC: H013-B308. AIAA Member.

** Sr. Research Engineer, Aeroacoustics Branch, MS 166. Sr. AIAA Member.
} 


$\begin{array}{ll}S P L & =\text { sound pressure level }=20 \log \left(p_{\text {rms }} / p_{\text {ref }}\right), p_{\text {ref }}=2 \cdot 10^{-5} \text { newton } / \mathrm{m}^{2} \\ \mathbf{T} & =\text { T-type chevrons } \\ T & =\text { tunnel noise } \\ t . e . & =\text { trailing edge } \\ T T R & =\text { total temperature ratio, core/fan } \\ V & =\text { flow velocity, convection velocity } \\ \mathbf{V} & =\text { flaperon with contra-rotating type of mini vortex generators } \\ \mathrm{vg} & =\text { vortex generator } \\ \mathbf{W} & =\text { flaperon with co-rotating or parallel type of mini vortex generators } \\ \beta & =\text { sweep angle } \\ \delta_{f l a p} & =\text { flap angle detent } \\ \Omega & =\text { vorticity }\end{array}$

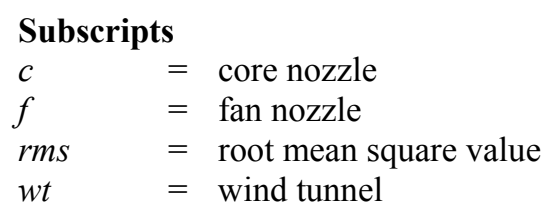

\section{Introduction}

GOR aircraft with engines under the wing and with engine exits upstream of the flaps, a complex interaction $\boldsymbol{\Gamma}$ occurs between the propulsion jet stream and the airframe which can change the far field total noise characteristics compared to the sum of the isolated jet noise and the isolated airframe noise (without the jet). For example, part of this propulsion/airframe aeroacoustic interaction can be due to the reflection of jet noise by the wings, or change in the evolution of the jet itself, or additional noise sources created by the flap edges being in the jet stream or its entrainment region, etc. The interaction between the jet and the flaps can be especially strong when the flaps are deployed, such as, at approach conditions making the noise sources at the flap edges more pronounced. This latter phenomenon is called as the "jet-flap interaction (JFI)" noise ${ }^{1}$ and its reduction gains importance as the noise rules for aircraft become more and more stringent, forcing us to look into incremental sources of noise reduction. In this paper, we study the effect on JFI due to modification of a type of flap called a "flaperon," which is, functionally speaking, a high speed aileron between the inboard and the outboard flaps.

Two important parameters for JFI noise are the flap/flaperon deflection angle and the jet velocity. During approach conditions the flaps are deployed considerably but the jet velocity is lower; whereas, at take-off conditions the flaps are not deployed as much but the jet velocity is much higher. When the flaperon also happens to be located right above the jet centerline, as in our study, JFI noise can be significant even if the flaperon is less deployed but the jet speed is higher, as at take-off. Hence, it is important to study JFI noise at both approach and take-off conditions although traditionally airframe/fan noise and jet/fan noise are considered dominant sources of noise at those two conditions respectively. ${ }^{2}$

Jet-flap interaction noise has been studied quite well in the literature and several original references can be found in Fink's review on "Propulsive Lift Noise." For under the wing configurations, Fink lists three mechanisms (see Fig. 8 in Ref. 1): (i) Lift fluctuation noise, (ii) Trailing edge noise, and (iii) Quadrupole noise from deflected jet (also known as impact noise). The common feature in all of them is that they radiate noise predominantly in the front quadrant due to the deflection of the flap independent of the type of singularity (dipole, quadrupole, etc.) representing the noise source. When the flaps/flaperons are not completely immersed in the jet stream, as is being considered in this paper, the JFI noise can only be considered as a weak form of propulsive lift noise. Although all the above mechanisms have some relevance to JFI noise, the most important one from the viewpoint of JFI reduction is the trailing edge noise mechanism where the convection of turbulent eddies across the trailing edge of the flap generates noise. Trailing edge noise has been studied quite thoroughly in the literature, as in the authoritative reviews by $\mathrm{Howe}^{3}$ and, later, by Crighton ${ }^{4}$. We use some of the scaling laws described in Crighton to reduce trailing edge noise and the concepts will be discussed in the next section.

In terms of experiments on jet-flap interaction noise useful to our study, we make note of the work in SenGupta ${ }^{5}$ and Brown/Ahuja ${ }^{6}$. SenGupta analyzes both jet-wing interaction noise and jet-flap interaction noise: the first one is defined when the flaps are not deflected and is modeled in terms of lift fluctuation noise, trailing edge noise, and jet noise reflection; the second one, JFI noise, is defined as the additional component purely due to flap deflection and is modeled in terms of lift fluctuations on the flaps diffracted by the wing trailing edge. The effect of engine strut 
height, flap cut-out (spanwise gap between flap segments around the engine exhaust) and porous extension to the trailing edge of flaps on JFI noise was studied parametrically and beneficial ways to reduce it were shown. Flap cutouts, when present around the engine exhaust, also bring out another important source of JFI noise when the flaps are deflected, namely, flap side-edge noise which is generated due to the unsteady rolling up of the tip vortex at the flap side edges. Brown/Ahuja attribute the mid-frequency hump in the front quadrant in their noise data to JFI noise, and study the effect on it due to a systematic variation of flap parameters, namely, flap angle, flap cut-out, angle of attack, location of jet with respect to the wing and jet velocity. Elkoby ${ }^{7}$ has studied the issue of propulsion airframe aeroacoustic (PAA) interactions, which includes JFI as a component, at full scale by examining the differences between flight test data and static engine test data projected to flight test conditions without the installation effects. These spectral differences at full scale were not always explainable although they matched the trends from scalemodel tests.

Although all the above studies are helpful in getting a general understanding of the phenomenon of PAA interactions and, in particular, jet flap interaction noise, they do not address the specific issue of reduction of JFI noise due to modification of flaperons for a modern aircraft whose engine may also be equipped with jet noise reduction devices. This leads to the objective of this paper: to study experimentally the effect of modifying the flaperon on the reduction in noise radiated in the far field region as well as at the source, that is, near the flaperon, in the presence of a modern coaxial jet at both approach and take-off conditions. Since the jet-flap interaction noise is also dependent on the shape of the velocity profile in the jet shear layer, we study the effect of modifying the flaperon not only in the presence of a simple round coaxial nozzle but also coaxial nozzles with jet noise reduction devices called "chevrons" (chevrons are unique triangular serrations on the nozzle lip known to reduce jet noise by affecting the shear layer). In this paper, we analyze the results for state-of-the-art azimuthally varying chevrons $(\mathrm{AVC})^{8,9}$ on the fan nozzle which have proven quieter than the conventional uniform chevrons. Some of the flaperon modifications are unique and have not been tried before, and the JFI noise study with both chevron nozzles and flaperon modifications is the first of its kind in the literature, to the authors' knowledge.

This paper is part of a larger study at Boeing on Propulsion Airframe Aeroacoustic (PAA) interactions, funded by NASA in 2004, and should be read in conjunction with the companion paper ${ }^{10}$ on the effect of AVC on JFI noise without the flaperon modifications. The scope of this work is limited to modern higher bypass ratio nozzles, of the order of 7 to 8 and the focus here is on noise, not thrust. Also, modifications to the flap side edge were out of the scope of this program.

\section{Flaperon Modification Concepts}

Broadly speaking, two types of modifications were tested: (i) sawtooth planform at the trailing edge of the flaperon, and (ii) mini-vortex generators (vg's) on the bottom/pressure side of the flaperon. The first is a fairly wellknown concept and the second one is new. Both these concepts are explained below.

\section{A. Sawtooth planform at the flaperon trailing edge}

It is well known that the far-field pressure fluctuations, $p_{r m s}^{2}$, due to the passage of turbulent eddies across a trailing edge of a half-plane are proportional to $\cos ^{3} \beta$, where $\beta$ is the angle between the normal to the trailing edge and the convection velocity of the eddies or the flow (see Figure 1, left side). When $\beta<<1$ the principal turbulent source contribution is referred to as the "principal edge noise dipole," the component normal to the half-plane of the acoustic dipole $Q=\Omega \times V$, where $\Omega$ is the flow vorticity vector and $V$ is the convection velocity. However, the scattered field induced by the interaction of this forcing dipole with the edge of the half-plane in not a dipole. More importantly, from the viewpoint of noise reduction, if the trailing edge is swept with respect to the flow, that is, $\beta \neq 0$, then this trailing edge noise component is lowered. Significant sweep of the whole flaperon edge is obviously not feasible, but one might hope that the same effect might be achieved by a sawtooth trailing edge, with alternating portions with large forward and backward sweep (see Figure 1, right side).

Crighton mentions that limited tests have been carried out on models with a sawtooth trailing edge where significant noise reductions of 3 to $6 \mathrm{~dB}$ were obtained for sweep angles of $60^{\circ}$ and full scale flight tests have also shown EPNL reductions of $2 \mathrm{~dB}$. In these cases, the beneficial sweep effect was along the whole length of the flap. It remains to be seen what part of it can be salvaged when the sawtooth edge is restricted to a smaller length, such as, that of the flaperon trailing edge span, especially, in the presence of the jet.

Serrated trailing edge can also be thought of as a means of obtaining a more gradual impedance change at the edge which would reduce noise. So far no noise reductions have been reported with such edges, though small noise increases have been reported. In light of these two observations, it seems that varying the geometrical parameters of 


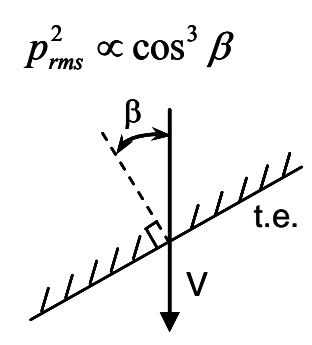

Swept trailing edge

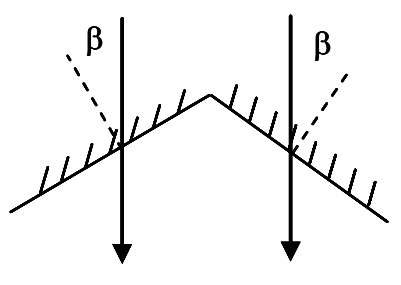

Sawtooth edge

Figure 1. Trailing edge (t.e.) noise dependence on trailing edge sweep angle, $\beta$, and sawtooth concept to reduce t.e. noise component.

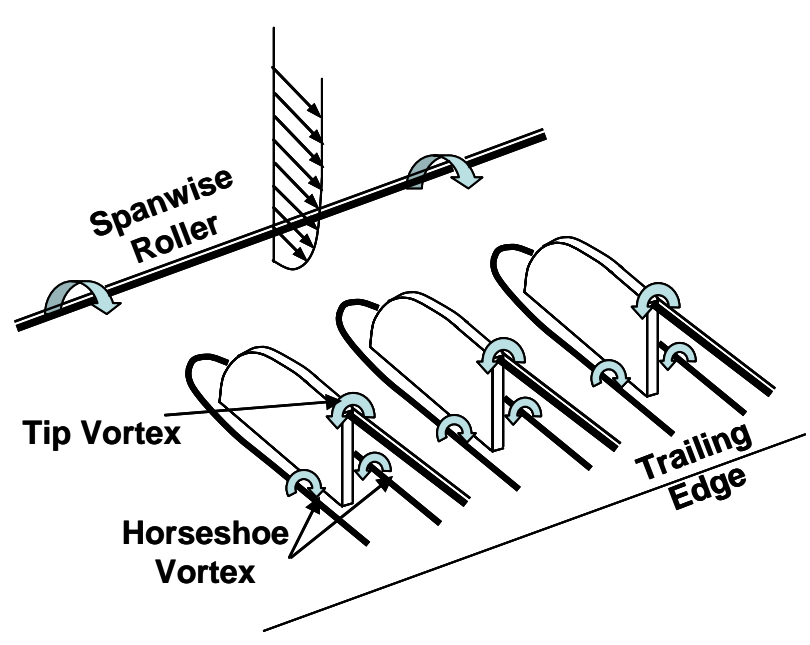

Figure 2. Tri-vortex array generation mechanism with parallel mini vg's of the W-type (tip vortex can change direction depending on the local angle of attack).

the sawtooth pattern, such as, its aspect ratio or sawtooth density per unit span are reasonable choices for exploration of JFI noise reduction.

\section{B. Mini Vortex Generators (vg's)}

As mentioned above, the principal edge noise dipole is strongest when the incident vorticity parallel to the edge is convecting normal to the edge with $\beta=0$. The boundary layer near the trailing edge is a source of such vorticity and if it can be distorted by some means then the spanwise coherence between it and the trailing edge will be lowered leading to a decrease in this noise component. In the previous concept we distorted the trailing edge by making it of sawtooth shape, but we can also distort the convecting vorticity itself so that the sweep angle, $\beta$, becomes closer to $90^{\circ}$. One way to do this is to have an array of flat plates perpendicular to the flaperon surface near its trailing edge (see Figure 2) so that this "spanwise roller" (with vorticity, $\Omega$ ) in the flaperon boundary layer can be bent around into several horseshoe vortices around the flat plates with the vorticity now becoming perpendicular to the trailing edge but still convecting downstream. This makes $\boldsymbol{\Omega}$ almost parallel to $V$, thus considerably decreasing the magnitude of the dipole $Q=\Omega \times V$ and, hence, lowering this noise component. This may be possible if the height of the plates is of the order of the boundary layer thickness near the trailing edge and not too much smaller than it.

However, in general the flow near the flaperon edge is quite complex and turbulent, and such coherent spanwise rollers may not exist under all operating conditions. The direction of convection velocities also may not be necessarily normal to the flaperon trailing edge. Hence, if we purposefully place this vertical plate at some angle of attack with the local flow then, as shown in Figure 2, its edge will generate another wing-tip type vortex whose direction will be approximately parallel to its convection velocity. An array of such plates will create an array of such tri-vortex system with vorticity convecting largely in the direction normal to the trailing edge. Depending on the angle of attack and the ratio of flat plate height to the local boundary layer thickness, it is possible that the horseshoe vortices may simply reduce to corner axial vortices of much weaker strength compared to the wing-tip vortex. We will call these plates mini-vortex generators (vg's). The primary idea here is to reduce the spanwise coherence of the convecting vorticity with the trailing edge thus reducing the trailing-edge noise component.

The idea of an array of such mini-vg's is not new by itself and has been used for a totally different purpose ${ }^{11}$ in aviation: when placed on top of wings (that is, on the suction surface) upstream of the ailerons, the array of vortices they ingest into the boundary layer can energize it allowing it to delay separation at higher angles of attacks and thus regaining some of the aileron control effectiveness.

In our application here for reduction of jet-flap interaction noise where the jet flow is on the bottom or pressure side of the flaperon, we will place the mini-vg's also on the bottom side of the flaperon rather than on the top or suction side. The top side of the flaperon sees the ambient flow which is at much less speed than the jet flow underneath it or the flow entrained by the jet stream, and at large deflection angles the ambient flow on the top side could also be separated thus rendering the mini-vg's useless. By placing them on the bottom/pressure side the hope 
is to manipulate the interaction of the jet/entrained flow with the trailing edge, and thus reduce JFI noise. The downside to this is that having placed the mini-vg's in a turbulent flow their leading edges can now act as efficient dipole noise radiators and can negate the noise benefit from the previous mechanism. It is still possible that there may be flow regimes where there may be net positive benefit for noise which needs to be explored.

Further, similar to the applications in aviation, as a parametric variation the mini-vg's can be placed either parallel to each other (same angle to the normal to the trailing edge) or in a zigzag fashion (say, plus/minus some angle to the normal to the trailing edge) so as to generate tip vortices of alternately different directions and render the spanwise vorticity incoherent with respect to the flaperon trailing edge in a yet different manner. These are called, respectively, co-rotating and contra-rotating mini-vg's.

\section{Scale Models and Experimental Setup}

The model scale flaperons were made to match the scale of the aircraft model which was already available at Boeing before this test program began. This is the same aircraft model previously used and described by the authors $^{9,10}$. Figure 3 shows some of the basic variations of the flaperon modifications that were tested. Their brief description is given below:

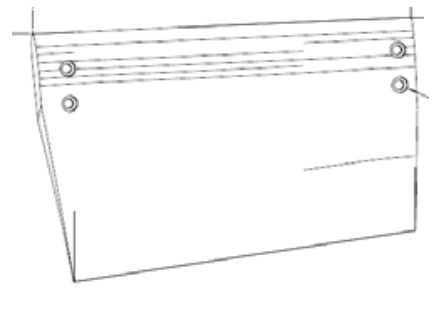

(a) Baseline, B

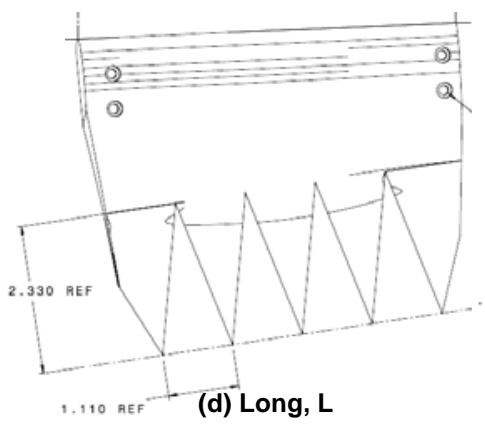

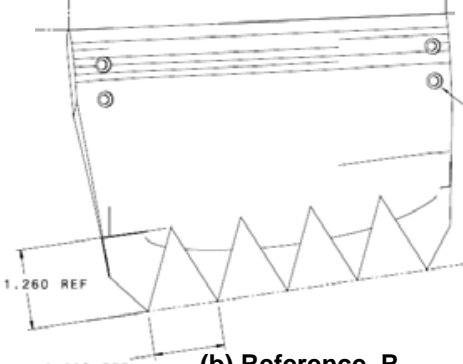

(b) Reference, $\mathbf{R}$

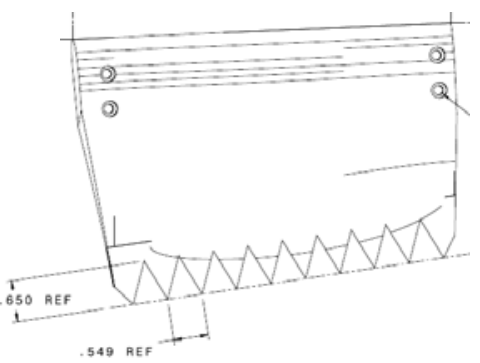

(e) Dense, $\mathbf{N}$

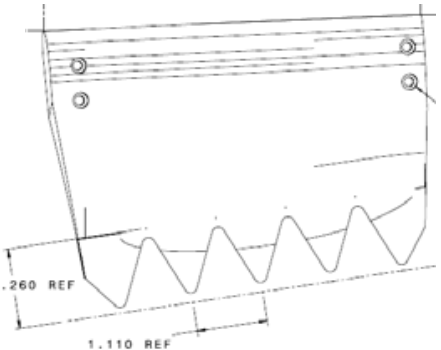

(c) Smooth, S

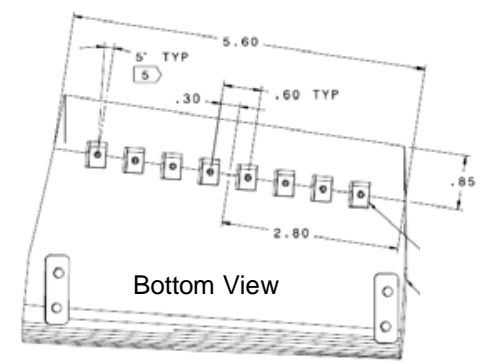

(f) Co-rotating mini-vg's, w

Figure 3. Some of the flaperon variations tested (top side shown on all, except for (f); all dimensions in inches).

a. Baseline flaperon, $\mathbf{B}$ : straight trailing edge

b. Reference sawtooth planform, R: 5 teeth, half in and half out of the baseline flaperon trailing edge to maintain its approximate area for aerodynamic purposes, aspect ratio $=1.135$, apex angle $=47.5^{\circ}, \beta=66.2^{\circ}$ (here $\beta$ is the angle between the normal to the sawtooth trailing edge (t.e.) and the normal to the locus of the tip line, which is parallel to the baseline flaperon's trailing edge; thus, if the flow were normal to the baseline flaperon's trailing edge then this would be the same sweep angle, $\beta$, as discussed in the trailing edge noise earlier; recall that the closer the angle $\beta$ is to $90^{\circ}$ the lower the t.e. dipole source strength)

c. Smoother sawtooth, $\mathbf{S}$ : tips and roots of $\mathrm{R}$ were rounded off thereby maintaining the apex angle and $\beta$ but reducing the aspect ratio somewhat; the idea was to reduce the high-frequency noise due to the sharp corners

d. Longer sawtooth, $\mathbf{L}$ : Higher aspect ratio (2.01 vs. 1.135) while maintaining the span of each sawtooth and their number as in $\mathrm{R}$, apex angle $=27.3^{\circ}, \beta=76.4^{\circ}$

e. Denser, $\mathbf{N}$ : Double the number of sawtooth compared to $\mathbf{R}$ (10 vs. 5) while maintaining approximately similar shape as $\mathbf{R}$, apex angle $=45.8^{\circ}, \beta=67.1^{\circ}$

f. Co-rotating mini-vg's, W: eight mini-vg's placed spanwise on the bottom side of the flaperon with (inter-vg spacing/distance from t.e.) $=0.71$, all at $5^{\circ}$ to the normal to the t.e. and parallel to each other; the details of 
the mini-vg's themselves are given in Figure 4 which shows an aspect ratio of 2 with a rounded leading edge facing the flow at the bottom of the flaperon.

A couple of variations of these flaperon configurations were also tested, important amongst them being the contra-rotating mini-vg's, $\mathbf{V}$, where the same elements were used as shown in Figure 4 and in the same geometrical pivot location as for $\mathbf{W}$-flaperon (see Figure 3), except that the mini-vg's were placed at $+/-5^{\circ}$ to the t.e. normal forming a zigzag pattern. The other variation was with the tips of the longer sawtooth rounded off, $\mathbf{L}$ '.

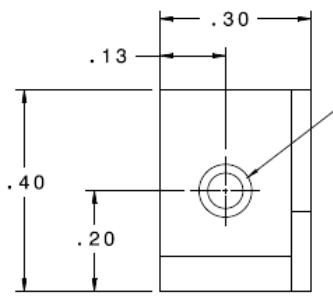

Top view

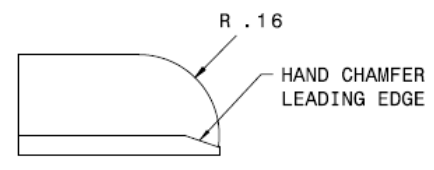

Left view

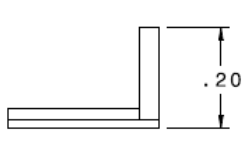

Front view

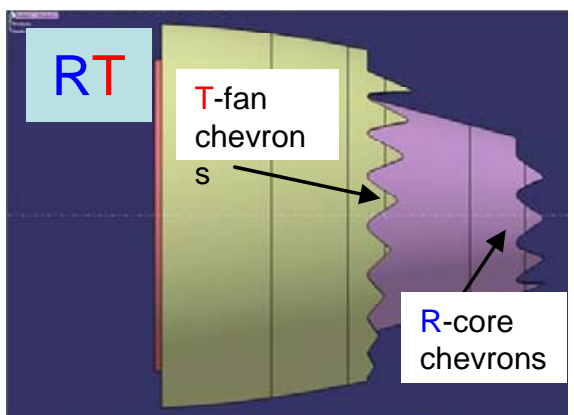

Figure 5. RT-chevron nozzle with azimuthally varying chevrons on the fan nozzle and regular uniform chevrons on the core nozzle.

Figure 4. Details of the mini-vortex generator (all dimensions in inches).

The baseline nozzle was an appropriately scaled round co-axial nozzle whose geometrical properties can be found in the authors' previous paper. Figure 5 shows the chevron nozzle, RT, whose test results are discussed in this paper. Its distinguishing feature is the azimuthally varying T-chevrons on the fan nozzle and the conventional uniform chevrons $\mathbf{R}$ on the core nozzle. Briefly, the $\mathbf{T}$-fan chevrons have longer and more immersed chevrons on the top near the pylon and smaller and less immersed chevrons at the bottom, diametrically away from the pylon. Further details on the RT-chevron nozzle can be found in the authors' previous papers ${ }^{8,9}$.

The test was conducted in Boeing's Low Speed Aeroacoustic Facility (LSAF) in Seattle and used the same general installed nozzle setup and instrumentation as described in some detail in Refs. 9 and 10. LSAF is a large anechoic chamber with a free jet in which the half-model is installed and immersed completely. Figure 6 shows the installed model with the left wing and the fuselage, both of which are supported by a bracket. Under the wing, and hidden from the view shown in Figure 6, is the scaled coaxial nozzle which is on a jet rig and attached to the wing through the pylon by a translating leak-proof joint. This coaxial nozzle has a hot air supply to simulate typical engine cycle conditions in the core and the fan nozzles.

The wing, as seen in Figure 6, has all its high-lift devices, such as, inboard and outboard flaps, flaperon and also the leading edge slats. Although the flap detents were changed depending on the cycle conditions (approach or takeoff), the slats were not deployed throughout the test for convenience. Figure 7 shows an aft view looking forward of the two flap deployments at approach and take-off when the flap detents were, respectively, $30^{\circ}$ and $5^{\circ}$. Notice that for this aircraft model (a) the centerline of the flaperon is on top of the engine exhaust centerline, (b) the inboard flap has slotted two-piece elements, and (c) the outboard flap is a one-piece element. During approach also notice that, especially, the side edge of the inboard flap is directly in the exhaust plume and would generate considerable noise by itself even though we are studying the effect of modifications of only the flaperon.

Two arrays of microphones were used and are described in detail in Refs. 8-10. We provide only a brief description here. The far field noise was measured by a polar array of microphones kept in a horizontal circular arc of radius $25 \mathrm{ft}$ centered around the core nozzle exit plane center with the core exit center in the same horizontal plane. The microphones spanned an angular range from $60^{\circ}$ to $150^{\circ}$ (measured from the nozzle inlet axis) and were directed towards the core exit plane center. We also used a phased array of microphones for source diagnostics purposes. These microphones were placed on a vertical plate and could be traversed horizontally on a track parallel 


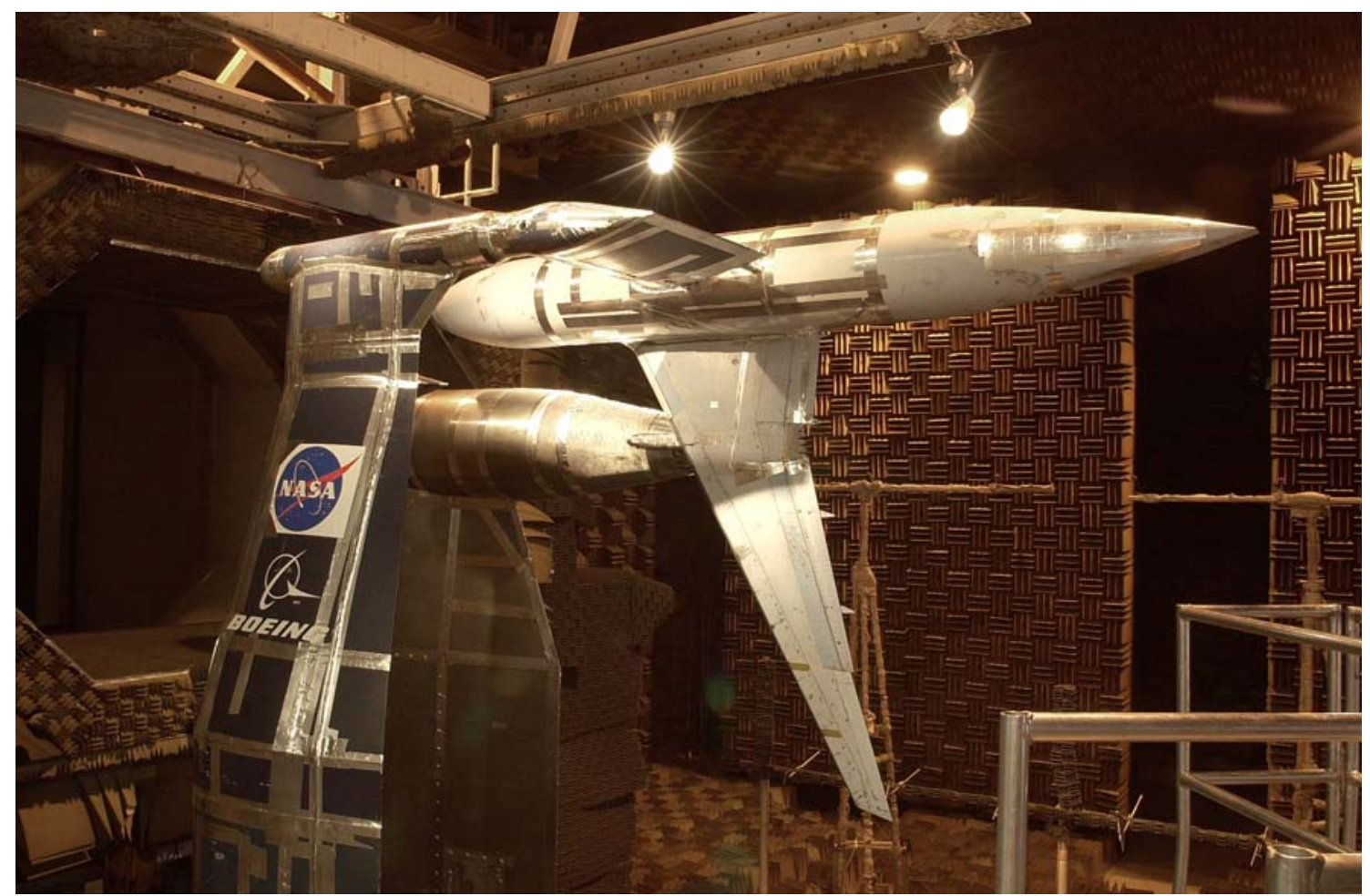

Figure 6. Installed scale-model setup in Boeing Low Speed Aeroacoustic Facility's jet noise rig to test the effect of flaperon modification in the presence of coaxial nozzles (modified long flaperon, $L$ ', shown).

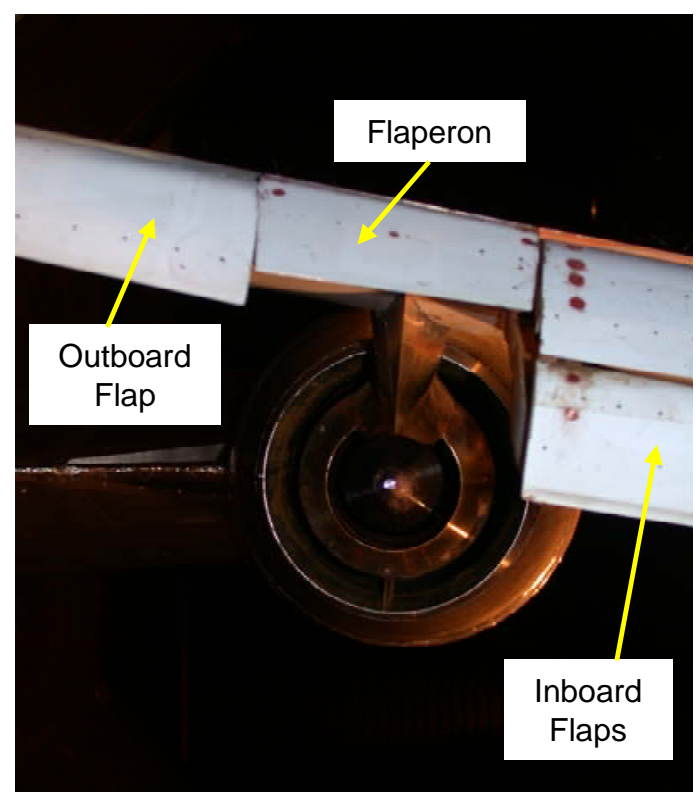

(a) Approach

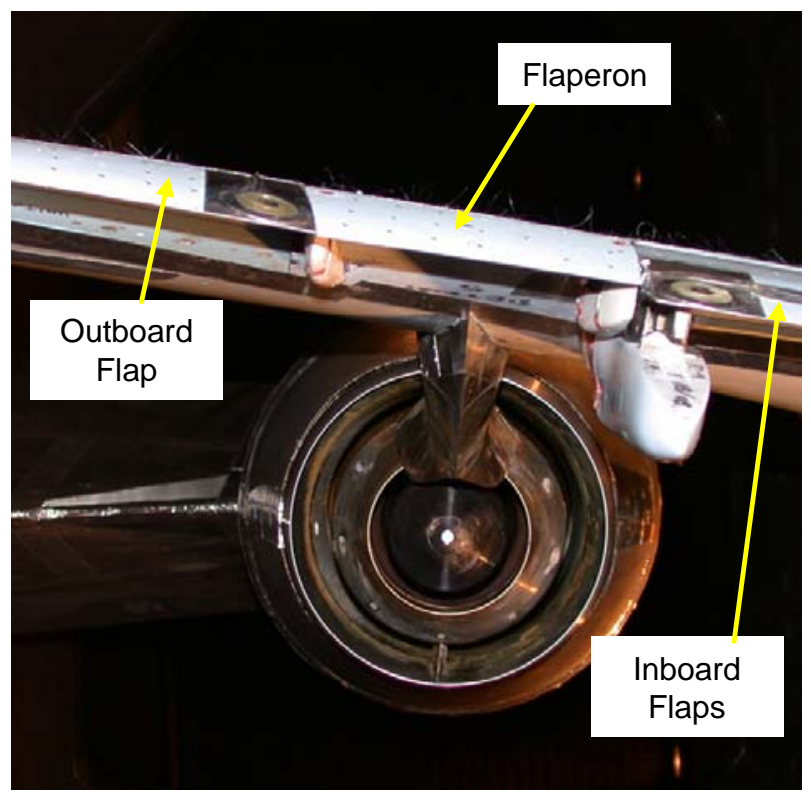

(b) Take-off

Figure 7. Comparison of flap deployments at approach $\left(30^{\circ}\right)$ and take-off $\left(5^{\circ}\right)$.

to the nozzle axis which was aligned with the windarray in relation to its location to the airplane model. For phased array was fixed such that the normal from the tunnel axis. Figure 8 shows a schematic of the phased the data analyzed in this paper, the location of the centroid of the array passed through the core exit plane 
center and the normal distance was $15 \mathrm{ft}$. Figure 8 also shows the relative location of the source plane mapping which will be used throughout this paper: it is parallel to the phased array vertical plane and passes through the

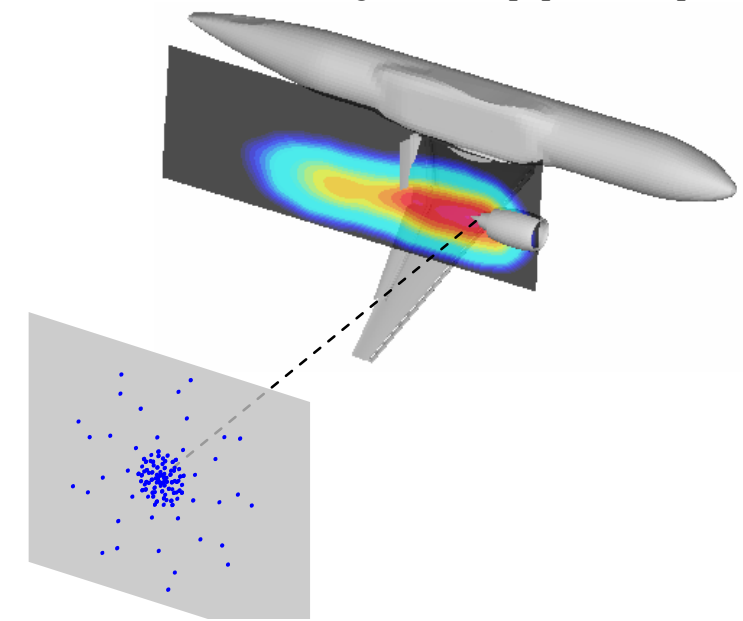

Figure 8. $90^{\circ}$-phased array location and source plane mapping relative to the airplane model. nozzle centerline. The noise data reported in this paper are the sound pressure levels (SPL) plotted on this source plane as measured by the phased array at the location of the phased array itself, not at the source plane location.

\section{Experimental Results and Discussion}

We will first analyze the test data for the effect of flaperon modifications in the presence of the baseline coaxial nozzle. This will be done initially for the approach condition when the flaps are at $30^{\circ}$ detent and then for the take-off condition at full power, also known as the sideline condition, when the flaps are at $5^{\circ}$ detent. We will try to corroborate part of the far field noise data with the phased array data in terms of origin and strength of the source changes. Then we will use the quieter flaperons, if any, and study them in conjunction with the azimuthally varying chevron nozzle at both the conditions.

The two cycle conditions analyzed here are the same

as in the authors' previous studies ${ }^{8-10}$ and are tabulated below:

\begin{tabular}{|c|c|c|c|c|c|}
\hline Condition & $\mathbf{N P R}_{\mathbf{f}}$ & $\mathbf{N P R}_{\mathbf{c}}$ & $\mathbf{T T R}$ & $\mathbf{M}_{\mathbf{w t}}$ & $\boldsymbol{\delta}_{\text {flap }}$ \\
\hline Approach & 1.218 & 1.114 & 2.096 & 0.24 & $30^{\mathbf{o}}$ \\
\hline Sideline Take-Off & 1.735 & 1.622 & 2.513 & 0.30 & $5^{\mathbf{o}}$ \\
\hline
\end{tabular}

\section{A. Data Repeatability and Tunnel Noise Floor}

Narrowband SPL was measured in LSAF from $200 \mathrm{~Hz}$ to $89 \mathrm{KHz}$ and converted to $1 / 3^{\text {rd }}$ octave band $\left(10^{*} \log (\right.$ frequency $\left.)\right)$ ranging from band number 22 to 49 . We have previously documented ${ }^{8,9}$ that during this LSAF test the repeatability of the far field SPL is within $\pm 0.5 \mathrm{~dB}$ between band levels of 27 and 47 at all directivity angles; it is even tighter $( \pm 0.3 \mathrm{~dB})$ if we restrict to a smaller band range ${ }^{8,9}$ around band 36 . The phased array results, on the other hand, are more accurate and have a smaller repeatability error of $\pm 0.15 \mathrm{~dB}$ throughout the frequency range of interest.

Before we begin the study of far field noise at approach conditions where the tunnel Mach number is 0.24 and the jet speeds are relatively lower, we first need to examine if the tunnel noise contaminates the far field noise. If it does contaminate the data then we need to see which ranges of frequencies and angles to avoid and which to include in our analysis. This issue was studied in detail in Fig. 6 of the companion paper where the spectra of tunnel noise was compared with the "airframe" noise (when the tunnel is on but the jet is off), and the "installed" jet noise (when both the tunnel and the nozzle jet flows are on in the presence of the airframe).

Figure 9(a) shows a contour plot of the SPL difference between the measured installed noise and the tunnel noise in the spectral-directivity plane - the higher the difference, the lesser the contamination due to tunnel noise. Thus, for example, the $10 \mathrm{~dB}$-contour plot (border of white-colored region) demarcates the band-angle region where the tunnel noise is $10 \mathrm{~dB}$ lower than the measured installed noise and the far field noise differences, say, due to flaperon modifications in that region ( $\geq 10 \mathrm{~dB}$ (white colored region)) can be considered to be statistically significant rather than due to tunnel noise fluctuations if they are also above the error band due to repeatability. Conversely, note from Fig. 9(a), that around band 35, where the repeatability error is also small $( \pm 0.3 \mathrm{~dB})$ as noted earlier, the tunnel noise is 8.5 to $9 \mathrm{~dB}$ lower than the measured installed noise in the "front" quadrant $\left(60^{\circ}\right.$ to $\left.90^{\circ}\right)$ and the SPL differences there due to flaperons can be considered to be statistically significant if they are also above the scatter band. This is, indeed, the band-angle region of interest for studying JFI noise.

Another point to be understood is the following: when we make measurements, say, of installed noise (with both the tunnel and the jet on) it is the total sum, $S$, of the tunnel noise, $T$, and the true installed noise, $I$, that is being measured ( $S, T$ and $I$ are in dB's). Since $I$ and $T$ are uncorrelated, $S$, is the $\log$ sum of these two sources. It is easy to relate the difference $(S-T)$ to the difference between the true installed noise and tunnel noise, $(I-T)$ : 


$$
I-T=10^{*} \log \left(10^{\frac{S-T}{10}}-1\right)
$$

Figure 9(b) shows this relationship between these two differences. Thus, for example, when S-T $=3 \mathrm{~dB}$, that is, when the measured installed noise is only $3 \mathrm{~dB}$ more than the tunnel noise, then $\mathrm{I}=\mathrm{T}$ or the true installed noise is equal to the tunnel noise which is not a desirable situation since I can go below $\mathrm{T}$ and be masked by $\mathrm{T}$. Hence, we need to restrict to those regions in the spectral-directivity plane which has (S-T) much larger than $3 \mathrm{~dB}$. Fig. 9(b) shows that when the total measured noise is, say, $8.5 \mathrm{~dB}$ above the tunnel noise then the true installed noise is approximately $8 \mathrm{~dB}$ above the tunnel noise and is considered here to be a reasonable difference to measure the true effects of flaperon modifications. Having said that, let us note that throughout this paper we are really interested in differences in SPL due to flaperon modifications, not the absolute SPL, in which case the tunnel noise, assumed to be the same for the two cases being compared, is not of much consequence so long as the installed noise is reasonably well above it. Thus, when analyzing far field SPL data, we will restrict to those regions in the band-angle plane where the tunnel noise is much lower than the measured installed noise and the SPL differences are larger than the error due to repeatability. It is also for this reason that we try to corroborate the far field SPL results with those from the phased microphone arrays in the band-angle regions of interest, when possible, because the crosscorrelation techniques used in the latter minimize the effect of tunnel noise and can pin-point the source of the far field SPL differences.

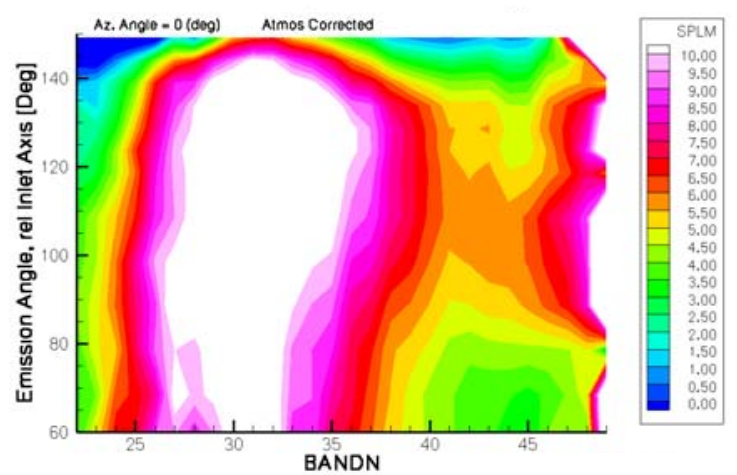

(a) Difference between measured installed noise and tunnel noise, $\mathrm{dB}$ (white color for $\geq 10 \mathrm{~dB}$ )

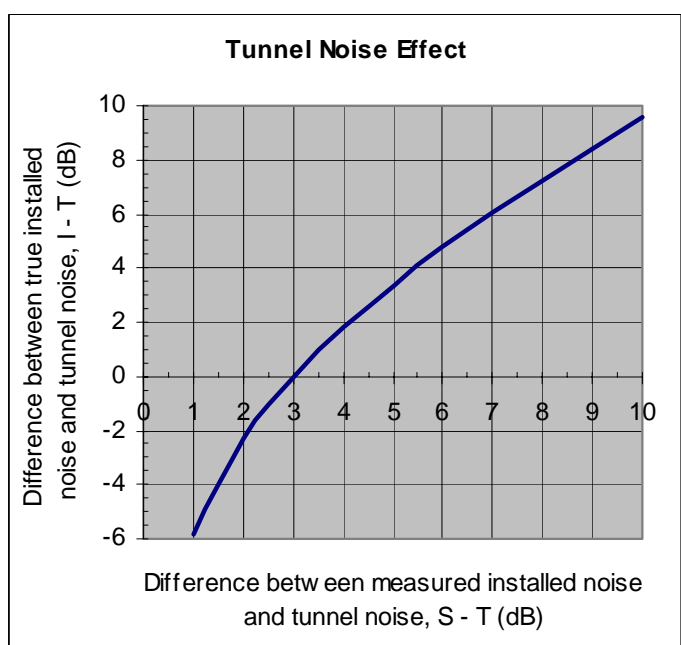

(b) Relationship between measured installed noise, true installed noise and tunnel noise

Figure 9. Region of influence of tunnel noise in the frequency-directivity plane at approach conditions and relationship between total measured installed noise, true installed noise and tunnel noise (tunnel Mach number $=0.24$ ).

\section{B. Effect of Flaperon Modifications with the Baseline Nozzle}

\section{Approach Conditions}

Figure 10 shows the far field SPL difference between the reference sawtooth flaperon, $\mathrm{R}$, and the baseline flaperon, B. A negative value of $\triangle \mathrm{SPL}$ is better (quieter) and a value beyond the repeatability error band will be considered a statistically significant effect. Thus, we see that there is a marginal beneficial effect up to $1 \mathrm{~dB}$ with the R-flaperon especially in the front quadrant at lower frequencies. Recall that earlier studies ${ }^{10,6}$ have denoted this region also as the one dominated by JFI. We will corroborate this later with phased array analysis.

Figure 11 shows the effect due to (a) longer serrations (higher aspect ratio), L, (b) denser serrations, N (c) contra-rotating mini-vg's, V, and (d) co-rotating mini-vg's, W. Flaperon L with sawtooth of higher aspect ratio of 2 appears to increase the noise, especially, at higher frequencies, and is worse than R (compare Figure 11(a) to Figure 10). The denser similar-shaped serrations, N, do not make much difference in noise. Both the mini-vg's, however, appear to be beneficial (dark green colored regions) over a bimodal range of angles (upstream and downstream) and 


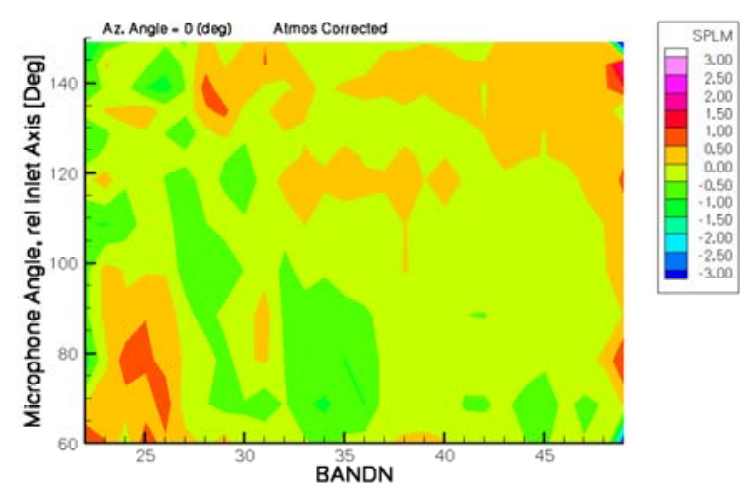

Figure 10. Difference in far field SPL (dB) in spectral-directivity plane between the reference sawtooth flaperon (R) and the baseline straightedged flaperon (B) at approach conditions for baseline nozzle.

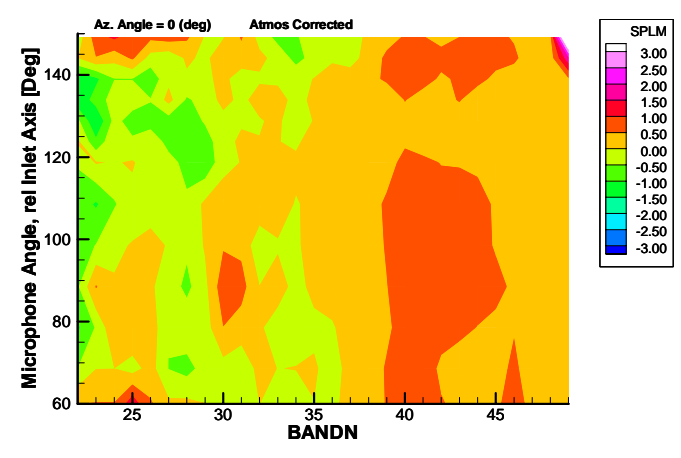

(a) Longer serrations (L)

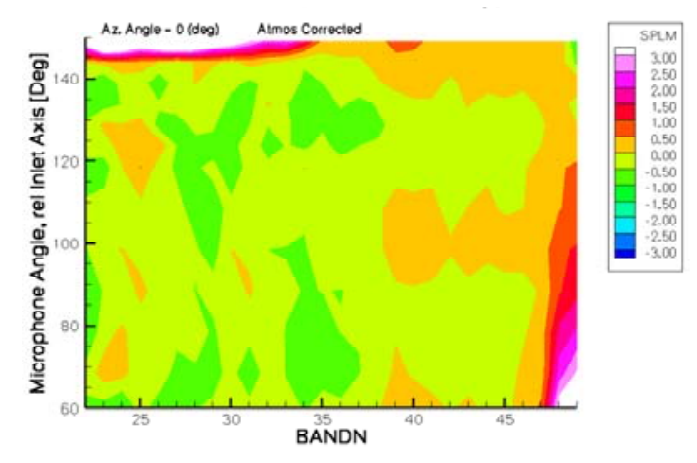

(c) V-type contra-rotating mini-vg's generally restricted to the low to medium frequencies. The co-rotating mini-vg's, W, appear to be more beneficial (SPL difference of $1+\mathrm{dB}$ ) especially in the downstream quadrant, near $130^{\circ}$, normally the region dominated by jet noise; it also shows benefit in the usual region dominated by JFI in the upstream quadrant near band 35 . Thus, the co-rotating mini-vg's, W, appear to reduce JFI noise as well as jet noise at approach conditions.

Let us see if we can corroborate some of these far field noise results with the source diagnostics tool. Figures 12(a-f) show the 90-degree source diagnostics maps for all these flaperons: $\mathrm{B}, \mathrm{R}, \mathrm{L}, \mathrm{N}, \mathrm{V}$ and $\mathrm{W}$, respectively, in terms of spatial SPL contour plots at four different frequencies, $1223 \mathrm{~Hz}, 2054 \mathrm{~Hz}, 6131 \mathrm{~Hz}$ and $12232 \mathrm{~Hz}$ corresponding to band numbers of $30.9,33.1$, 37.9 and 40.8 . If the source is downstream of the core exit plane then the line of sight going through the centroid of the $90^{\circ}$-phased array points in the upstream quadrant and this remains true even after accounting for

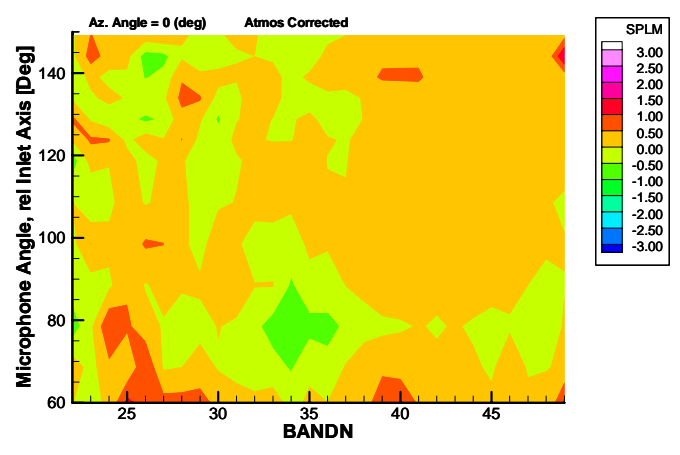

(b) More number of serrations (N)

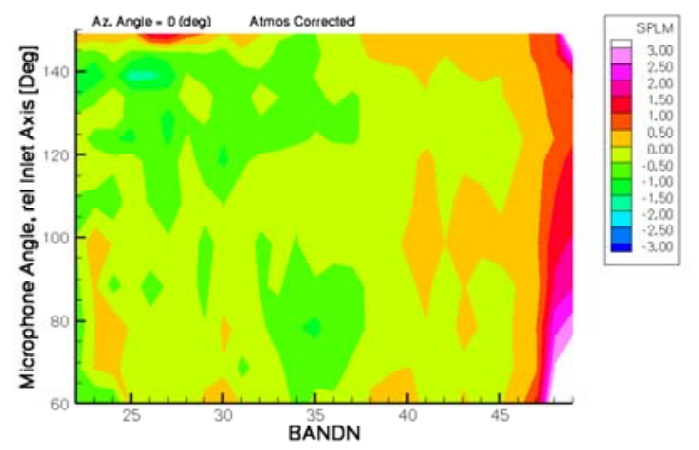

(d) W-type co-rotating mini-vg's

Figure 11. Flaperon modification effect on far field sound at approach conditions - difference in SPL (dB) between the modified flaperon ( $L, N, V$ or $W$ ) and the baseline straight-edged flaperon (B) for a baseline simple round coaxial nozzle. 
(a) Baseline Flaperon, B
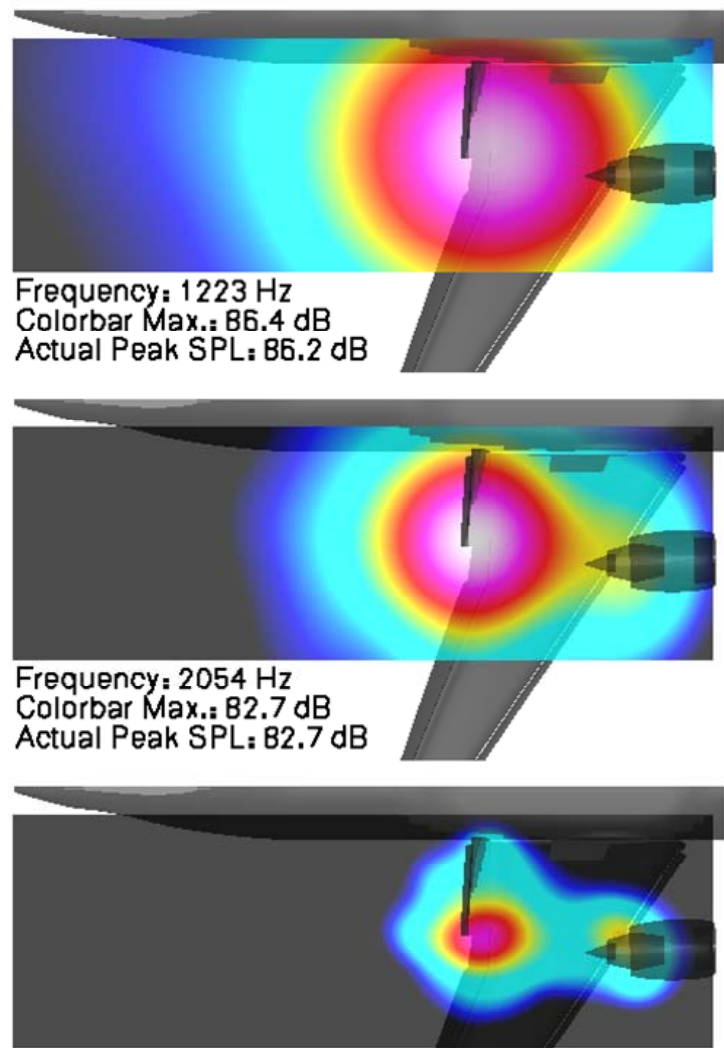

Frequency: $6131 \mathrm{~Hz}$ Colorbar Max.: $74 \mathrm{~dB}$ Actual Peak SPL: $72.8 \mathrm{~dB}$

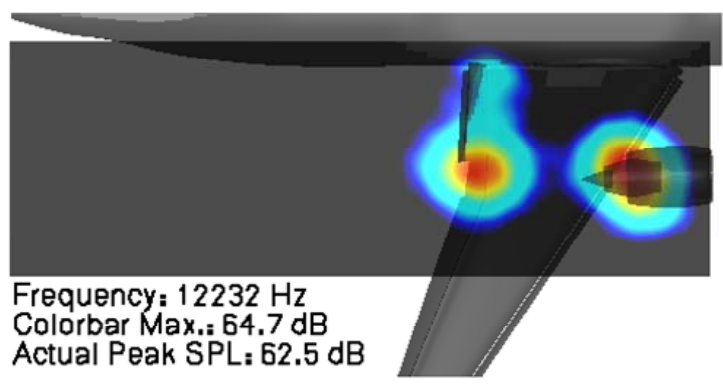

(b) Reference Sawtooth Flaperon, R
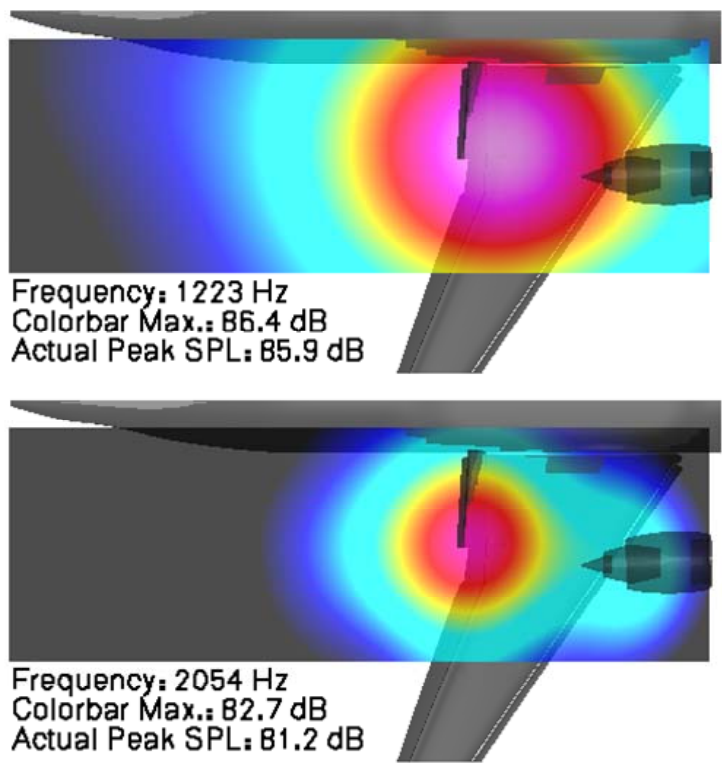

Colorbar Max.: $82.7 \mathrm{~dB}$

Actual Peak SPL: B1.2 dB

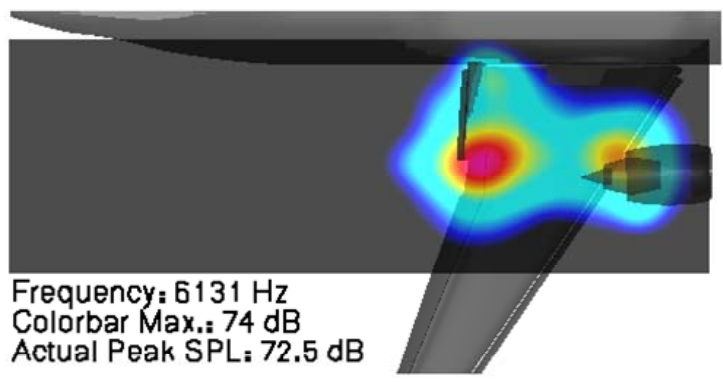

Colorbar Max.: $74 \mathrm{~dB}$
Actual Peak SPL: $72.5 \mathrm{~dB}$

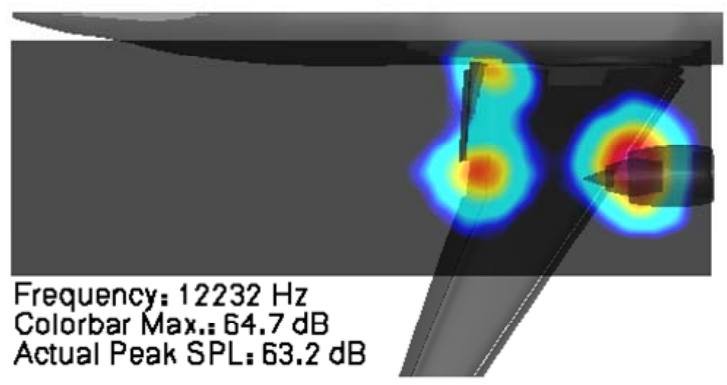

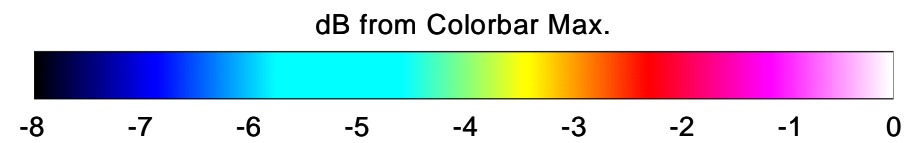

Figure 12. Flaperon modification effect at the source with the baseline nozzle for different frequencies at approach conditions as seen by the phased array at $90^{\circ}$ : (a) baseline straight-edged flaperon, B and (b) the reference sawtooth flaperon, $R$. 


\section{(c) Longer Sawtooth Flaperon, L}
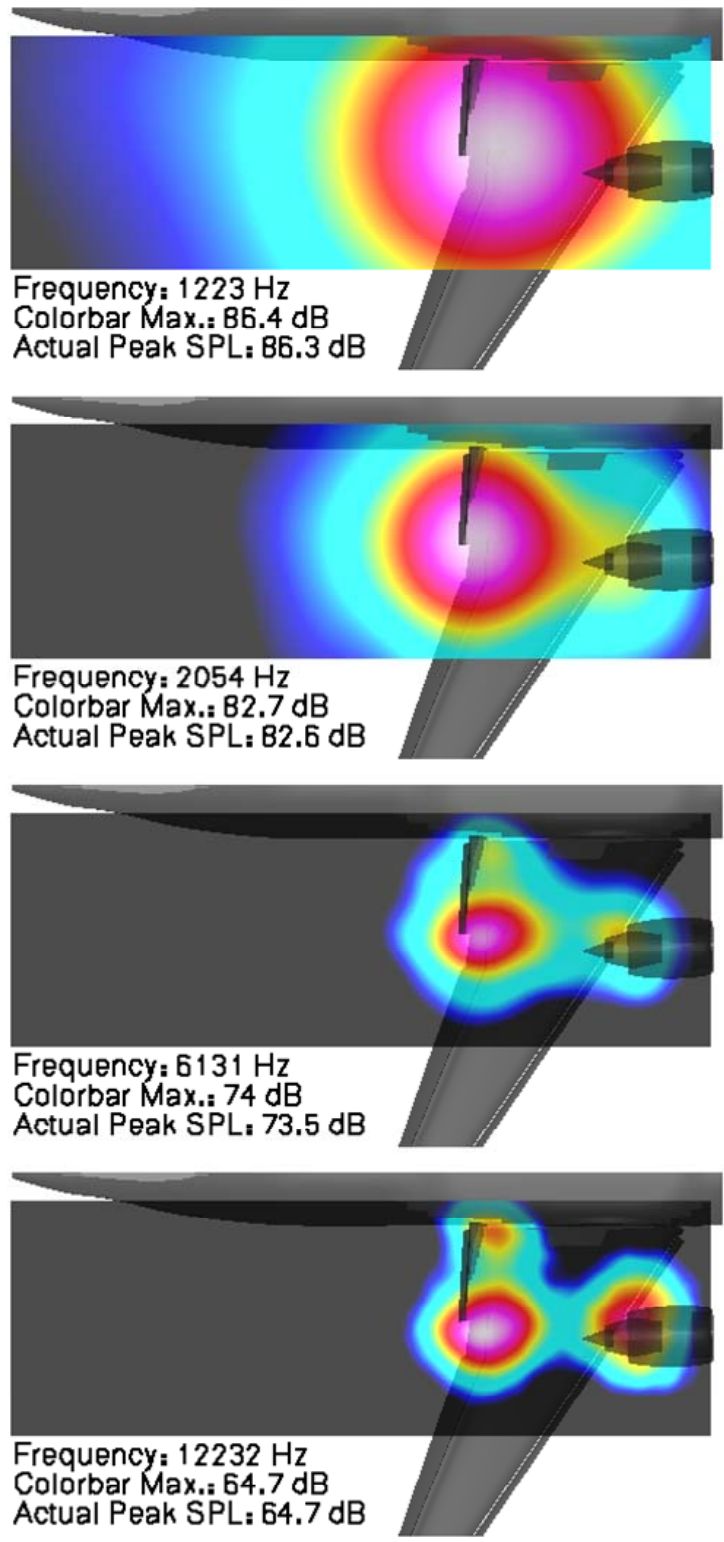

(d) Flaperon with More Number of Sawteeth, N
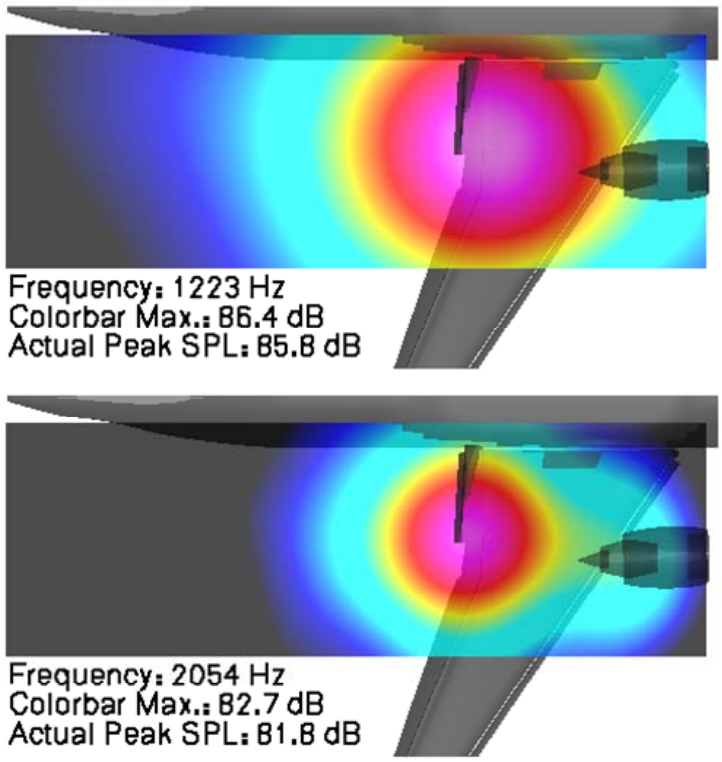

Colorbar Max.: $82.7 \mathrm{~dB}$

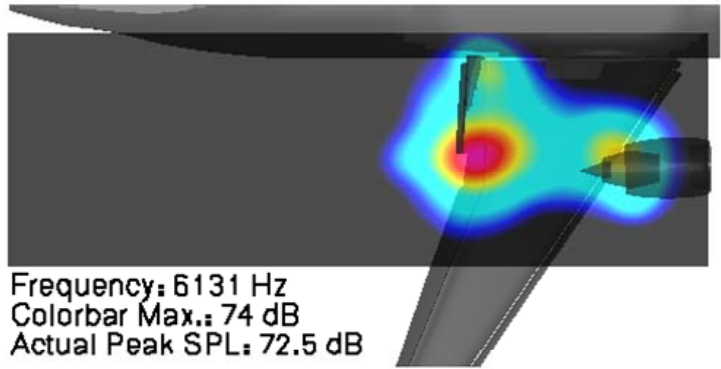

Colorbar Max : $74 \mathrm{~dB}$

Actual Peak SPL: $72.5 \mathrm{~dB}$

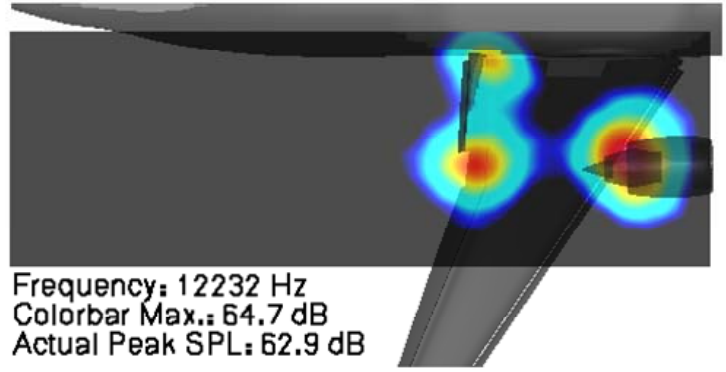

dB from Colorbar Max.

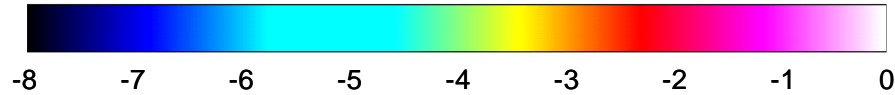

Figure 12 (cont'd). Flaperon modification effect at the source with the baseline nozzle for different frequencies at approach conditions as seen by the phased array at $90^{\circ}$ : (c) longer sawtooth flaperon, L, (d) flaperon with more number of sawteeth, $\mathrm{N}$. 

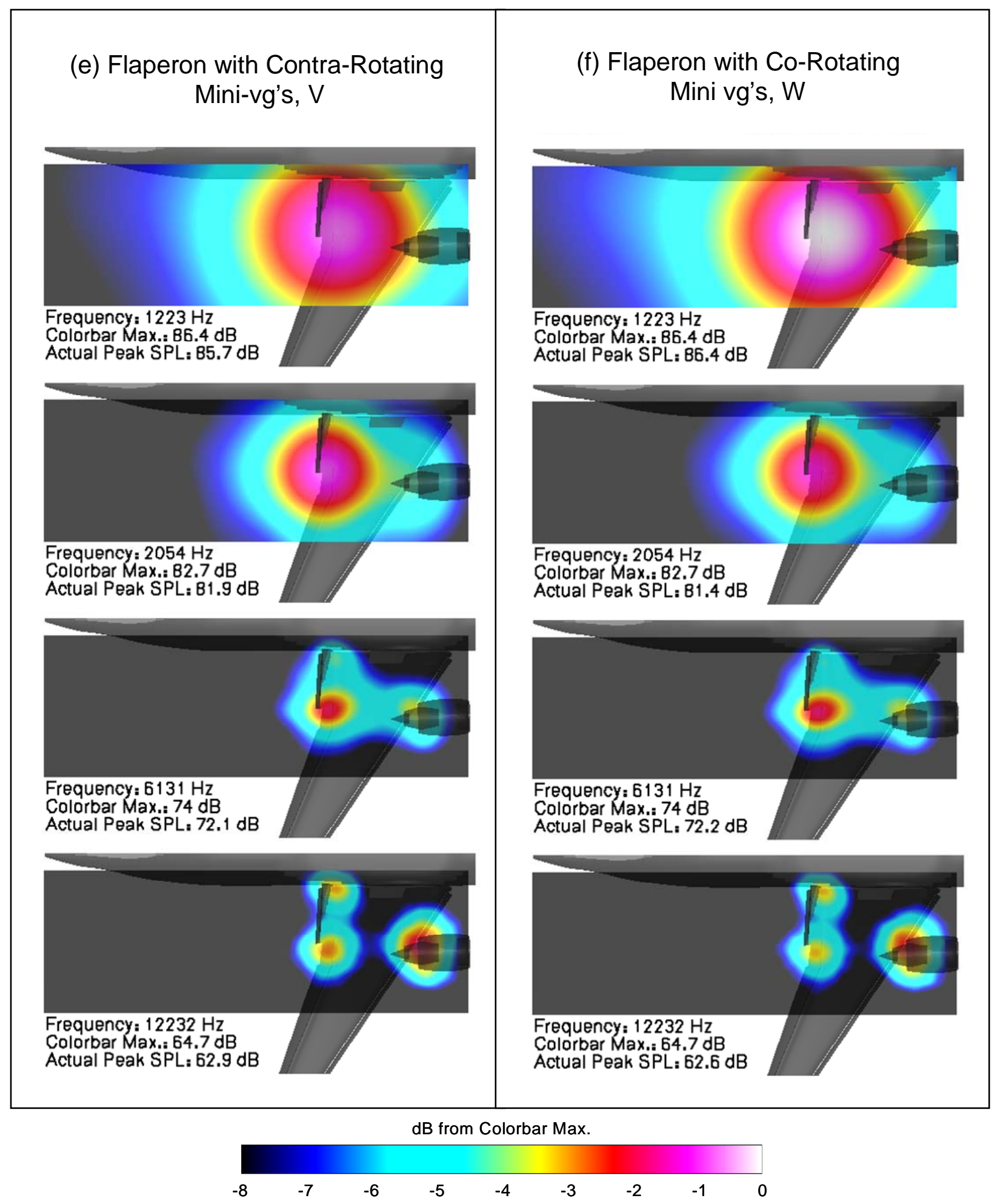

Figure 12 (cont'd). Flaperon modification effect at the source with the baseline nozzle for different frequencies at approach conditions as seen by the phased array at $90^{\circ}$ : (e) Contra-rotating mini-vg's, $\mathrm{V}$, (f) Co-rotating mini-vg's, W. 
the refraction at the tunnel shear layer. (See Ref. 10 for more details on this; it shows how the line of sight from the flaperon t.e. center to the centroid of the $90^{\circ}$-phased array makes an angle of $81.8^{\circ}$, and with the inclusion of tunnel flow refraction it corresponds to far field angles near $75^{\circ}$ which is, indeed, the region of interest for JFI.) Earlier we have seen that the upstream quadrant at these bands (30 through 41) is in a region where the tunnel noise does not contaminate the data. [Also note that in Fig. 12, the colorbar maximum value is held same for a given frequency for all flaperons, but that maximum value can change with frequency. The actual peak value is also denoted at the bottom of each plot, and the SPL difference colorbar scheme, common to all, is given at the bottom.]

From Figure 12(a) for the baseline flaperon, B, it appears that at lower frequencies the dominant source is located near the flaperon trailing edge, and as the frequency increases the spatial spread and the peak strength of this source decreases, and another source near the nozzle appears. By comparing the installed noise with the source diagnostics of isolated jet noise at the same low speed at approach conditions (high Strouhal number), we have shown earlier that the above source location downstream of the nozzle near the flaperon t.e. is not due to the jetalone contribution, but is truly due to the jet-flap interaction noise. Let us see the effect of some of the flaperon modifications:

- Comparison of R-flaperon with B-flaperon at $2054 \mathrm{~Hz}$ (Fig. 12(b) vs. Fig. 12(a)) shows that R has reduced the peak source strength near the flaperon by $1.5 \mathrm{~dB}$. This corroborates the far field SPL reduction in the upstream quadrant at that band, 33.1 (see Fig. 10). A small peak source strength reduction at other frequencies is also indicated.

- Comparison of L-flaperon with B-flaperon (Fig. 12(c) vs. Fig. 12(a)) at the higher frequencies, $12232 \mathrm{~Hz}$ and $6131 \mathrm{~Hz}$, shows an increase in the flaperon peak source strength of $2.2 \mathrm{~dB}$ and $0.6 \mathrm{~dB}$, respectively, thus corroborating the far field noise increase for $\mathrm{L}$ in the upstream quadrant (Fig. 11(a)).

- The denser sawtooth, N, (Fig. 12(d)) shows some reduction in the peak source strength at $6131 \mathrm{~Hz}$, and again corroborates the far field SPL reductions (Fig. 11(b)) around $80^{\circ}$ near band 33.1.

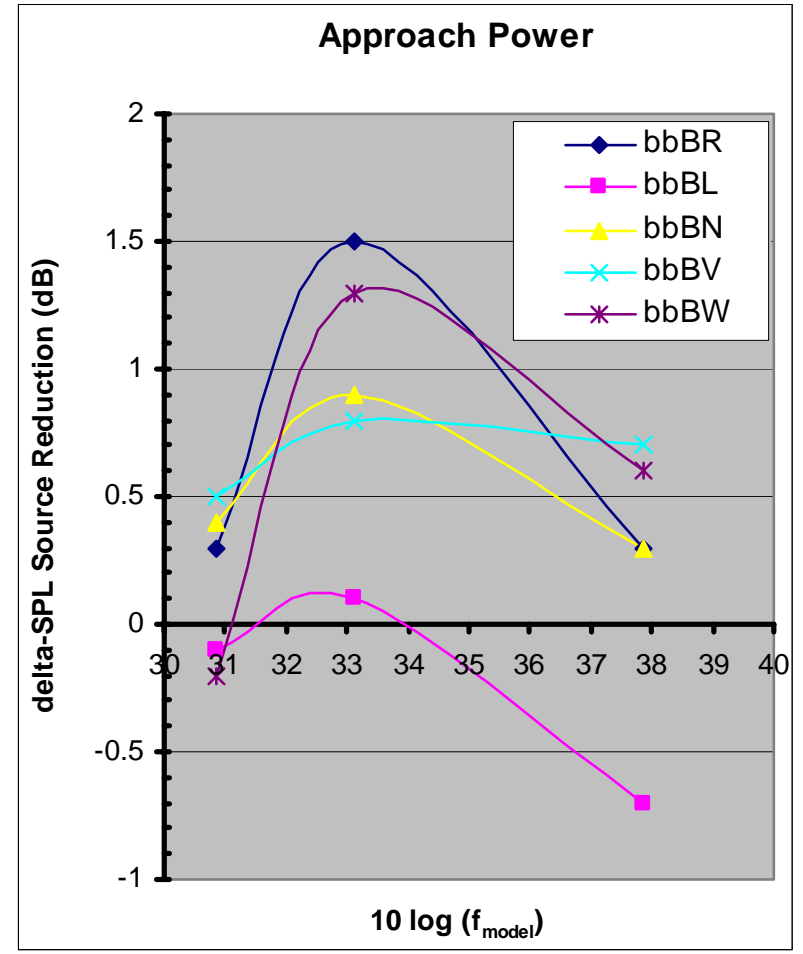

Figure 13. Effect of flaperon modifications (R, L, N, $V$ and $W$ ) on jet/flap interaction peak source noise reduction at different frequencies for baseline nozzle at approach power conditions compared to baseline nozzle with baseline flaperon, $B$, as seen by $\mathbf{9 0}^{\circ}$-phased array. (bbBx stands for baseline nozzle (bbB) and $x$ for the flaperon type)
- The contra-rotating mini-vg's, V, (Fig. 12(e)) generally show a peak source strength reduction near the flaperon t.e. of the order of 0.5 to $0.7 \mathrm{~dB}$ for the lower frequencies shown and a general reduction in the spatial distribution also which corroborates the far field SPL reduction (Fig. 11(c)).

- The co-rotating mini-vg's, W, (Fig. 12(f)), on the other hand, show a maximum peak source reduction at the flaperon t.e. of $1.3 \mathrm{~dB}$ at 2054 $\mathrm{Hz}$ and somewhat lower reductions at other frequencies, thus corroborating the far field SPL reduction results in the upstream quadrant for the mid-frequency bands. Unfortunately, the $90^{\circ}$-phased array cannot be used to corroborate the origin of the far field SPL reductions in the aft quadrant for the $\mathrm{W}$ flaperon (see Fig. 11(d)) since it is not in its line of sight for any sources downstream of the nozzle exit plane.

Thus, in general, we can conclude that (a) the phased array results corroborate the far field results for the different flaperon modifications quite well, and (b) the changes in the upstream far field noise due to the flaperon modifications, at least in specific bands (30 through 41), appear to originate at the flaperon t.e. due to JFI noise changes.

Figure 13 shows a summary of the peak source strength reductions in JFI noise due to the various flaperon modifications as seen by the $90^{\circ}$ phased array for the first three bands: 31,33 and 38 . These are above the error band of $0.15 \mathrm{~dB}$ and, hence, are statistically significantly reductions which affect the 
upstream quadrant in the far field. This shows that the reference sawtooth and the co-rotating mini-vg's provide the highest JFI source reductions (of the order of $1.5 \mathrm{~dB}$ ) at least for this upstream quadrant under approach conditions. Since these sources can be directive we cannot extend this observation to aft angles.

\section{Take-off conditions}

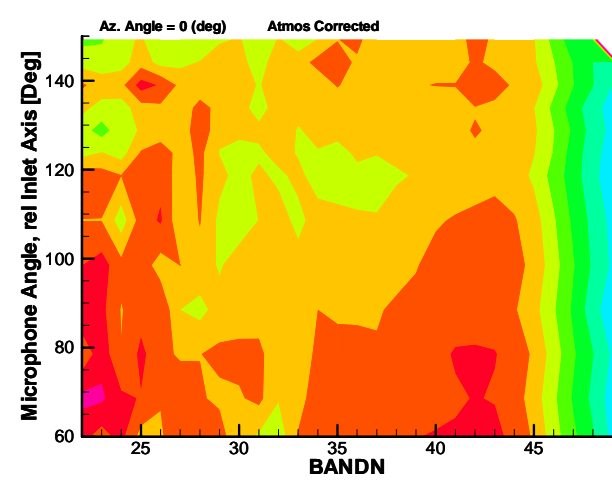

(a) Reference serrations, $\mathrm{R}$
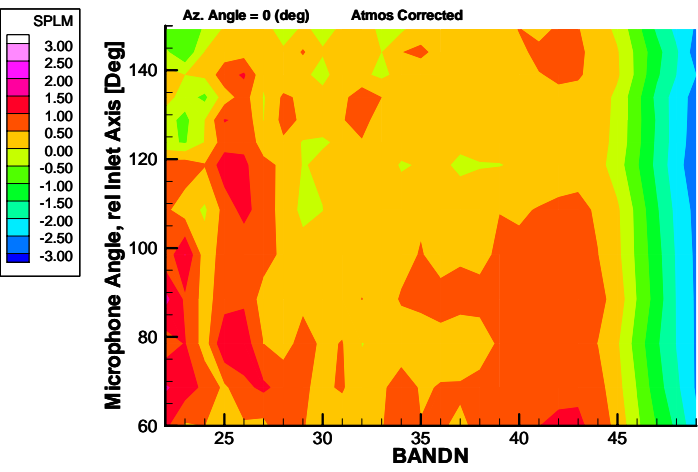

(b) Co-rotating mini-vg's, W

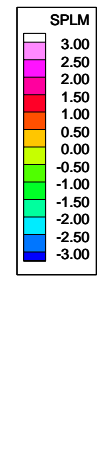

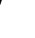

\section{Figure 14. Effect of flaperon modifications at sideline take-off power conditions for baseline nozzle (difference in SPL with respect to baseline flaperon is shown).}

Figure 14 shows the difference in far field noise due to the R- and $\mathrm{W}$-flaperons as compared to the baseline flaperon B at sideline take-off conditions for the baseline round nozzle. Both appear to increase noise everywhere, especially in the upstream quadrant for the low and high frequencies. This is exactly opposite to what happened under approach conditions.

Figure 15 shows the $90^{\circ}$-phased array plots for the baseline flaperon, B, and the co-rotating mini-vg's, W. For the baseline flaperon, B, comparison with isolated jet noise source location done earlier at this take-off power condition again shows that the peak source captured in Fig. 15(a) near the flaperon t.e. is not due to the jet plume alone but is, indeed, the true JFI noise location; the peak source location for the jet-alone is further downstream at these high speeds. The increase in noise due to $\mathrm{W}$ in the upstream quadrant appears to originate from the region near the flaperon t.e. where the mini-vg's are located (see Fig. 3(f)). It is also interesting to note that at $6131 \mathrm{~Hz}$ or band 38 the region of increased noise is also slightly more wide-spread from the nozzle exit to the flaperon t.e.

Thus, it appears that at high jet speeds of full take-off power, even with lower flap deflections, the mini-vg's introduce more noise when used in conjunction with the baseline round coaxial nozzle. It is possible that the leading edge dipole-type of noise of the mini-vg's, referred to earlier, is compensating for any possible noise reductions. Hence, the co-rotating mini-vg's, although helpful in reducing noise slightly at approach, are not useful at sideline conditions.

\section{Flaperon Modification Effects with Chevron Nozzle}

Due to the better success of the co-rotating mini-vg's with the baseline nozzle at approach conditions, we will study them in conjunction with the RT-chevron nozzle. Figure 16 shows the far field SPL difference between this RT-W nozzle-flaperon combination and the RT nozzle with baseline flaperon combination, RT-B, at both (a) approach and (b) sideline conditions. At approach, RT-W is noisier and at sideline it does not make much difference (within the data scatter limits).

Figure 17 for the B- and the $\mathrm{W}$-flaperons with the RT-nozzle confirms that the noise increase at approach, at least in the upstream quadrant, originates at the deployed flaperon t.e. and is due to the adverse jet-flap interaction between the RT-nozzle and the W mini-vg's. This appears to be true at all frequencies captured in Fig. 17 - both the peak source strengths and the region of influence at the flaperon t.e. are higher and larger for RT-W than for the RTB case. Figure 16 shows that this adverse JFI extends even to the aft quadrant in a very substantial way implying that either the mini-vg's are themselves radiating more noise in the aft direction or that they adversely affect the jet structure downstream. It is possible that this adverse interaction between the RT-nozzle flow and the W mini-vg's is due to the immersion of the mini-vg's into the top shear layer from the T-fan nozzle which is known ${ }^{12}$ to have enhanced mixing and larger spreading angle. 

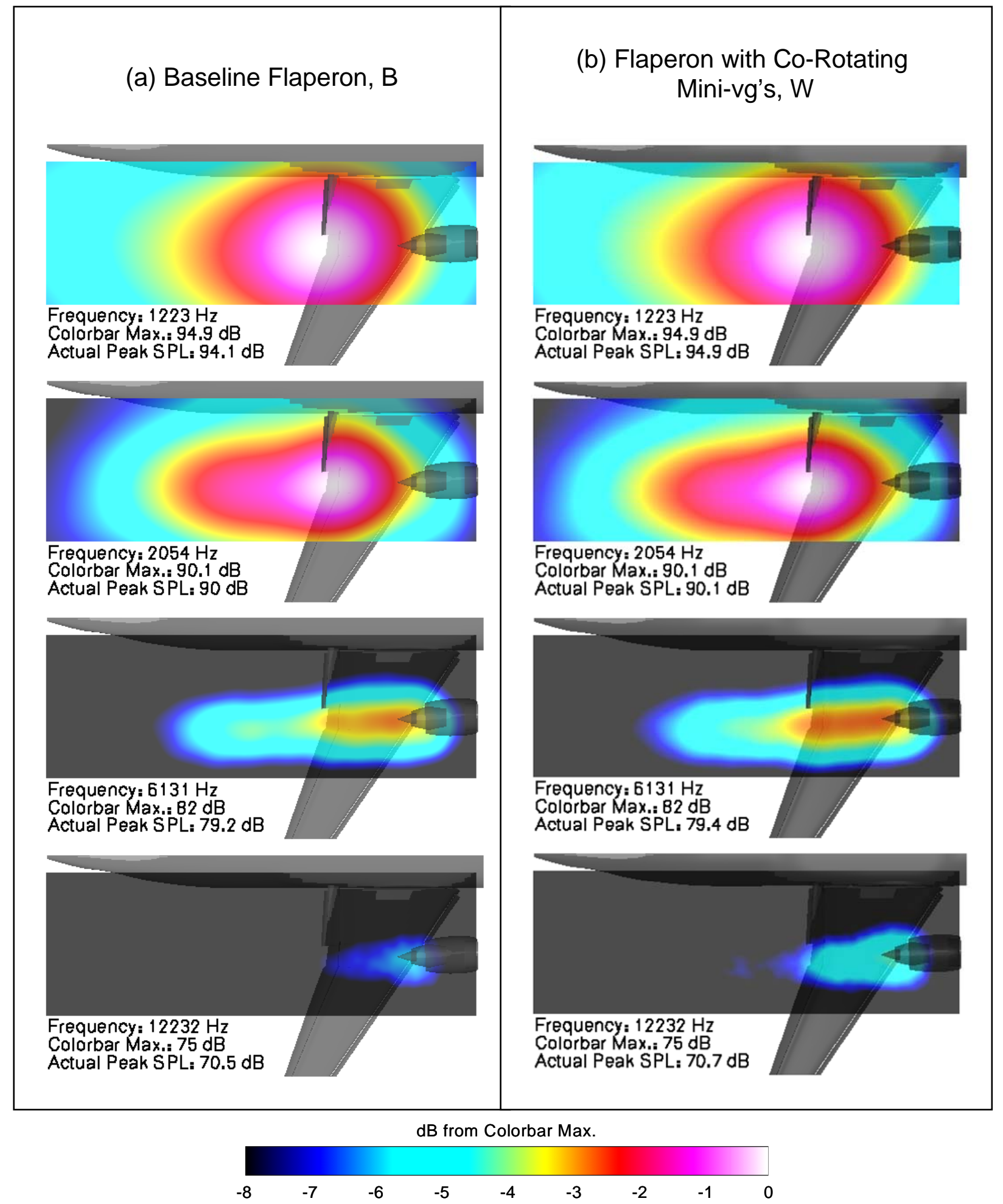

Figure 15. Flaperon modification effect at the source with the baseline nozzle for different frequencies at sideline take-off power conditions as seen by the phased array at $90^{\circ}$ : (a) Baseline flaperon, B, (b) Corotating mini-vg's, $W$. 


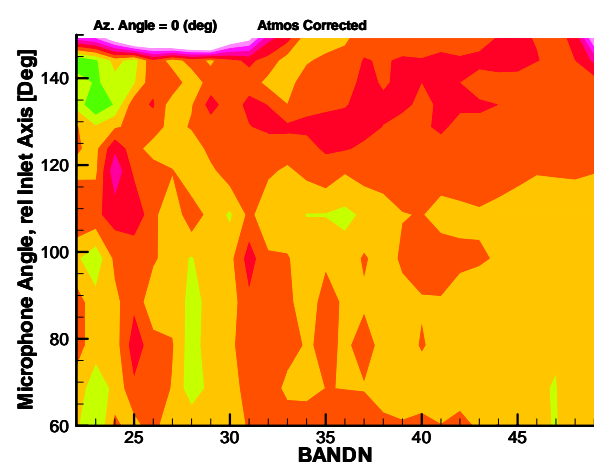

(a) Approach
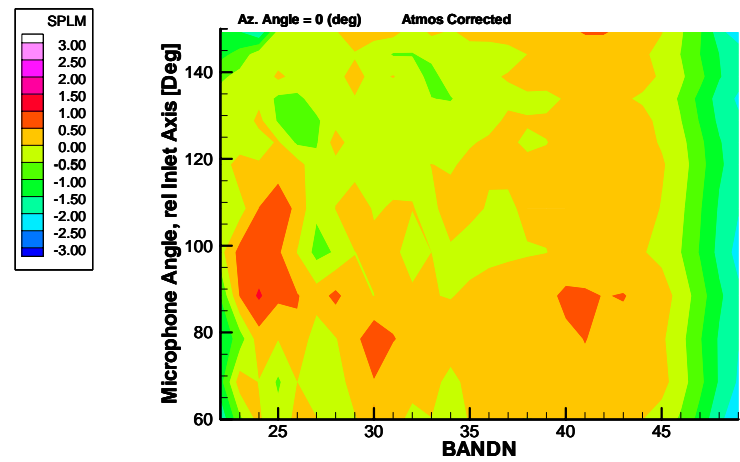

(b) Sideline

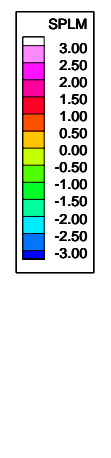
Figure 16. Effect of co-rotating mini-vg's, W, for RT-chevron nozzle at (a) approach and (b) sideline
take-off power conditions - difference in far field SPL spectral-directivity between modified flaperon and baseline flaperon is plotted.

Figure 18 shows the source strengths for the same cases at sideline conditions. The increase in JFI source strength at $1223 \mathrm{~Hz}$ due to W mini-vg's seems to corroborate the increase in the far field noise (see Fig. 16(b)) in upstream quadrant for the corresponding band 31. At moderate frequencies there is not much change in the source strength at the flaperon t.e., and at even higher frequencies, Fig. 18 shows that the peak source is away from the flaperon t.e. and is near the nozzle lip, as is usually the case at high jet speeds.

Thus, again, the phased array results corroborate well the far field noise changes due to the RT-W configuration. However, a deeper study is needed to understand the complex flow/acoustic interactions that take place between the advanced RT-chevron nozzle and mini-vg's. In general, it appears that the mini-vg's are not useful in conjunction with the RT-nozzle at either approach or sideline conditions.

\section{Conclusion}

The effect of two types of flaperon modifications, namely, sawtooth trailing edges and mini-vortex generators, on jet-flap interaction noise was studied for round coaxial nozzles and advanced chevron nozzles at both approach and take-off conditions. For the baseline round nozzle at approach conditions, the reference sawtooth trailing edge was marginally beneficial in reducing the far field noise in the front quadrant, and the co-rotating mini-vg's were slightly more beneficial in reducing the noise by about $1 \mathrm{~dB}$ over a wider angular range (both front and aft quadrants). The phased array results, indeed, pointed to the flaperon modifications as the sources of these reductions in the upstream quadrant. However, these two flaperon modifications were not useful at take-off conditions. Hence, the co-rotating mini-vg's on the flaperon can be helpful for round nozzles only if they can be deployed at approach and somehow removed at take-off, say, by folding/pulling them inwards. But the cost of doing this may not be worth the effort. For the advanced RT-chevron nozzle, the co-rotating mini-vg's caused an adverse JFI and are not deemed useful.

\section{Acknowledgments}

This work was done in 2004 under NASA Contract NAS1-00086. Boeing sincerely acknowledges the funding support given by NASA for this Propulsion Airframe Aeroacoustics project. We would also like to acknowledge the excellent support provided by the whole LSAF group during the test and, in particular, Steve Underbrink, Donn Perkins and Jim Underbrink. 

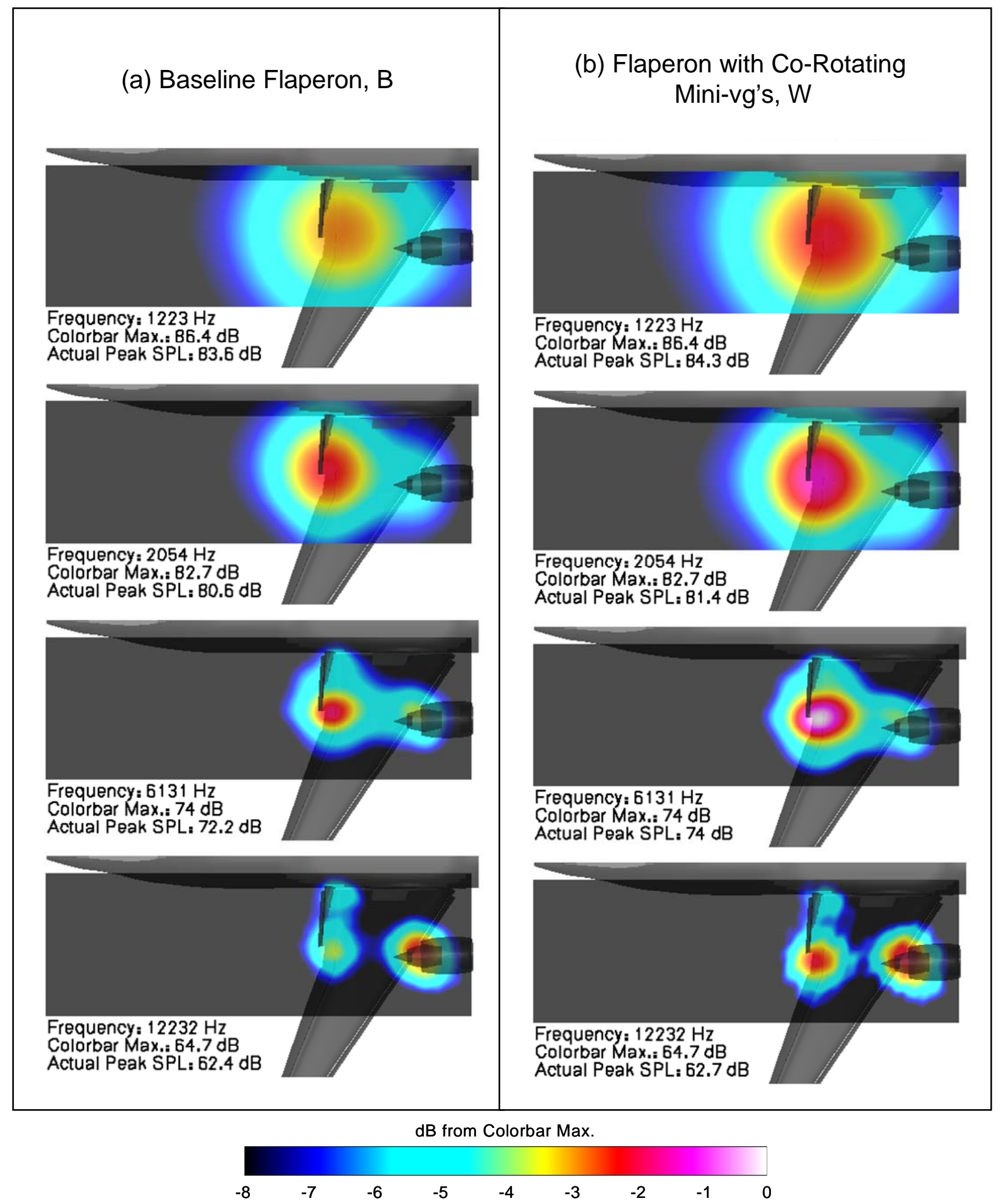

Figure 17. Flaperon modification effect at the source with the RT-nozzle for different frequencies at approach power conditions as seen by the phased array at $90^{\circ}$ : (a) Baseline flaperon, B, (b) Co-rotating mini-vg's, W. 

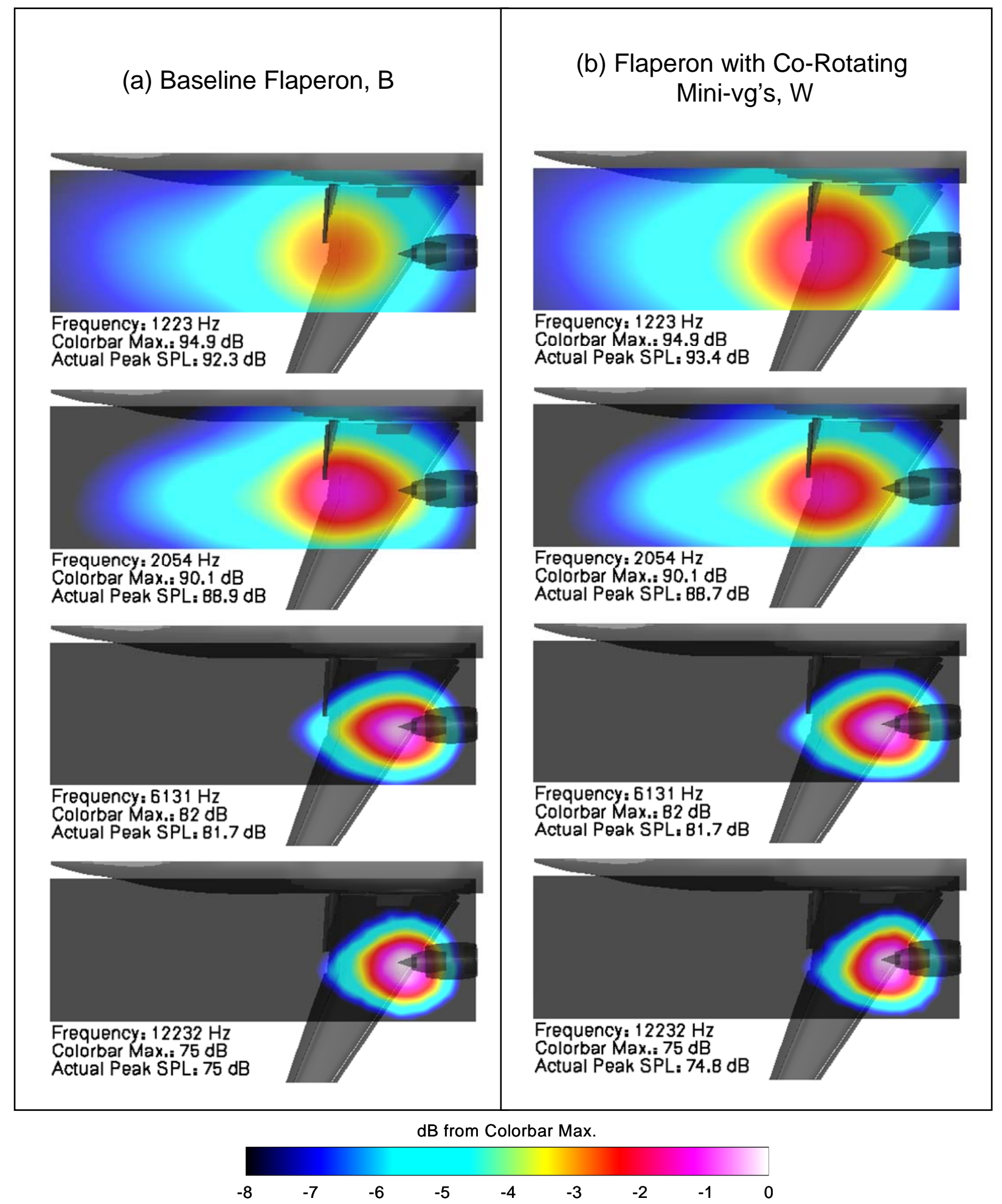

Figure 18. Flaperon modification effect at the source with the RT-nozzle for different frequencies at sideline take-off power conditions as seen by the phased array at $90^{\circ}$ : (a) Baseline flaperon, B, (b) Corotating mini-vg's, W. 


\section{References}

${ }^{1}$ Fink, M. R., "Propulsive Lift Noise," Aeroacoustics of Flight Vehicles: Theory and Practice, Volume 1: Noise Sources, edited by Hubbard, H.H., NASA Ref. Publication 1258, Vol. 1, Ch. 8, Aug. 1991.

${ }^{2}$ Groeneweg, J.F., Sofrin, T.G., Rice, E.J. and Gliebe, P.R., "Turbomachinery Noise," Aeroacoustics of Flight Vehicles: Theory and Practice, Volume 1: Noise Sources, edited by Hubbard, NASA Ref. Publication 1258, Vol. 1, Ch. 3, Aug. 1991.

${ }^{3}$ Howe, M.S., "A Review of the Theory of Trailing Edge Noise," J. Sound \& Vibration, vol. 61, no. 3, Dec. 8, 1978, pp. 437465

${ }^{4}$ Crighton, D.G., “Airframe Noise,” Aeroacoustics of Flight Vehicles: Theory and Practice, Volume 1: Noise Sources, edited by Hubbard, H.H., NASA Ref. Publication 1258, Vol. 1, Ch. 7, Aug. 1991.

${ }^{5}$ SenGupta, G., “Analysis of Jet-Airframe Interaction Noise,” AIAA Paper 83-0783, 1983.

${ }^{6}$ Brown, W.H. and Ahuja, K.K., "Jet and Wing/Flap Interaction Noise," AIAA Paper 84-2362, 1984.

${ }^{7}$ Elkoby, R., "Full-Scale Propulsion Airframe Aeroacoustics Investigation,” AIAA Paper-2005-2807, 2005.

${ }^{8}$ Mengle, V. G., Elkoby, R., Brusniak, L. and Thomas, R., "Reducing Propulsion Airframe Aeroacoustic Interactions with Uniquely Tailored Chevrons: 1. Isolated Nozzles,” AIAA Paper 2006-2467, 2006.

${ }^{9}$ Mengle, V. G., Elkoby, R., Brusniak, L. and Thomas, R., "Reducing Propulsion Airframe Aeroacoustic Interactions with Uniquely Tailored Chevrons: 2. Installed Nozzles," AIAA Paper 2006-2434, 2006.

${ }^{10}$ Mengle, V. G., Brusniak, L., Elkoby, R., and Thomas, R., "Reducing Propulsion Airframe Aeroacoustic Interactions with Uniquely Tailored Chevrons: 3. Jet-Flap Interaction,” AIAA Paper 2006-2435, 2006.

${ }^{11}$ Whitford, R., Design for Air Combat, Janes Publishing Co. Ltd., UK, 1987, pp. 108-109.

12 Massey, S.J, Elmiligui, A.A., Hunter, C.A., Thomas, R.H., Pao, S.P. and Mengle, V.G., "Computational Analysis of a Chevron Nozzle Uniquely Tailored for Propulsion Airframe Aeroacoustics,” AIAA Paper 2006-2436, 2006. 